\title{
A Generalized Fuzzy Integer Programming Approach for Environmental Management under Uncertainty
}

\author{
Y. R. Fan, ${ }^{1}$ G. H. Huang, ${ }^{1,2}$ K. Huang, ${ }^{1}$ L. Jin, ${ }^{3}$ and M. Q. Suo ${ }^{4}$ \\ ${ }^{1}$ Faculty of Engineering and Applied Science, University of Regina, Regina, SK, Canada S4S 0A2 \\ ${ }^{2}$ MOE Key Laboratory of Regional Energy Systems Optimization, Resources and Environmental Research Academy, \\ North China Electric Power University, Beijing 102206, China \\ ${ }^{3}$ College of Environmental Science and Engineering, Xiamen University of Technology, Xiamen, Fujian 361024, China \\ ${ }^{4}$ College of Urban Construction, Hebei University of Engineering, Handan, Hebei 056038, China
}

Correspondence should be addressed to G. H. Huang; huangg@iseis.org

Received 10 March 2014; Revised 6 June 2014; Accepted 17 June 2014; Published 14 September 2014

Academic Editor: Hang Xu

Copyright (c) 2014 Y. R. Fan et al. This is an open access article distributed under the Creative Commons Attribution License, which permits unrestricted use, distribution, and reproduction in any medium, provided the original work is properly cited.

In this study, a generalized fuzzy integer programming (GFIP) method is developed for planning waste allocation and facility expansion under uncertainty. The developed method can (i) deal with uncertainties expressed as fuzzy sets with known membership functions regardless of the shapes (linear or nonlinear) of these membership functions, (ii) allow uncertainties to be directly communicated into the optimization process and the resulting solutions, and (iii) reflect dynamics in terms of waste-flow allocation and facility-capacity expansion. A stepwise interactive algorithm (SIA) is proposed to solve the GFIP problem and generate solutions expressed as fuzzy sets. The procedures of the SIA method include (i) discretizing the membership function grade of fuzzy parameters into a set of $\alpha$-cut levels; (ii) converting the GFIP problem into an inexact mixed-integer linear programming (IMILP) problem under each $\alpha$-cut level; (iii) solving the IMILP problem through an interactive algorithm; and (iv) approximating the membership function for decision variables through statistical regression methods. The developed GFIP method is applied to a municipal solid waste (MSW) management problem to facilitate decision making on waste flow allocation and waste-treatment facilities expansion. The results, which are expressed as discrete or continuous fuzzy sets, can help identify desired alternatives for managing MSW under uncertainty.

\section{Introduction}

Municipal solid waste (MSW) management is a priority for many developed and developing countries throughout the world. Effective planning of MSW is critical for supporting sustainable socioeconomic development in urban communities. However, extensive uncertainties may exist in many system components and impact factors. For example, waste generation rate within a city is related to many socioeconomic and environmental factors and exhibits various uncertain features. Such uncertainties and their interactions can lead to increased complexities in the related planning efforts and will affect consequent decision processes. Besides, these uncertainties may be further multiplied because many system components are of multiperiod, multilayer, and multiobjective features Li and Huang [1]. Moreover, waste-disposal facilities in a MSW management system usually have overall cumulative or daily operating-capacity limits. Increasing waste generation rates, as a result of population explosion and economic development, lead to intensified conflicts with decreasing waste-treatment/disposal capacities. Therefore, it is desired that the above uncertain and dynamic complexities be reflected in efforts for identifying effective environmental management alternatives.

In the past decades, a number of inexact optimization techniques were developed to deal with uncertainties and dynamics in MSW management. They were mainly classified as fuzzy, stochastic, and interval mathematical programming (FMP, SMP, and IMP, resp.) [2-6]. For example, Li and Huang [7] proposed an inexact two-stage mixed-integer linear programming (ITSMILP) method for solid waste management in the city of Regina, through incorporating 
interval linear programming (ILP), two-stage stochastic programming (TSP), and mixed-integer programming (MIP) within a general mathematical programming framework. However, IMP could merely deal with interval uncertainties without distributional information; SMP was inapplicable to large-scale problems due to its stringent requirements for information of probabilistic distributions.

Fuzzy mathematical programming (FMP), as a branch of fuzzy set theory, could generally deal with uncertainties expressed as fuzzy sets or fuzzy goals/constraints [8-11]. Recently, various FMP methods were employed to deal with uncertainties in MSW systems [12-15]. For example, Fan et al. [12] explored a fuzzy linear programming (FLP) method for dealing with uncertainties expressed as fuzzy sets that exist in the constraints' left-hand and right-hand sides and the objective function; however, this method was unable to reflect dynamic complexities related to capacity expansion schemes for waste-treatment facilities. Srivastava and Nema [13] proposed a fuzzy parametric programming model for identifying desired treatment/disposal facilities, planning waste management capacities, and allocating waste flows under uncertainty; however, the proposed method generated deterministic waste allocation schemes without provision of bases for supporting generation of multiple decision options corresponding to the uncertain system conditions.

Generalized fuzzy linear programming (GFLP) (or fully fuzzy linear programming (FFLP)) methods extended traditional FMP approaches through permitting uncertain information in the optimization process and resulting solutions. Recently, several GFLP (or FFLP) methods were proposed to deal with uncertain information in both parameters and decision variables [16-21]. For example, Hosseinzadeh Lotfi et al. [18] developed a lexicography method to solve the FFLP problem and generate approximate solutions presented as fuzzy sets. However, previous studies on fuzzy variables in FMP problems mainly focused on some special types of fuzzy sets (such as symmetric, triangular, or trapezoidal fuzzy sets). Furthermore, some of them might lead to complicated intermediate models and thus were not applicable for large-scale problems. Fan et al. $[19,20]$ proposed another kind of fuzzy programming (named generalized fuzzy linear programming (GFLP) method) to deal with fuzzy uncertainty in both parameters and variables, in which all fuzzy sets with known membership functions can be treated through defuzzification method (i.e., $\alpha$-cut method). However, the GFLP approach cannot reflect dynamic features in environmental management problems. Moreover, no previous study was reported on capacity expansion issues under fuzzy conditions through the generalized fuzzy optimization approach, where expansion schemes were desired under multiple scenarios and $\alpha$-cut levels.

As an extension of developed GFLP approach, a generalized fuzzy integer programming (GFIP) method would be proposed for MSW management under uncertainty. The proposed GFIP approach integrates the techniques of generalized fuzzy linear programming (GFLP) and mixed-integer programming (MIP) within an optimization framework. In detail, (i) the GFIP method can deal with uncertainties expressed as fuzzy sets with known membership functions, regardless of whether these functions are linear or nonlinear; (ii) the proposed GFIP method can allow uncertainties to be directly communicated into the optimization process and the resulting solutions; (iii) the GFIP method can reflect dynamics in terms of waste-flow allocation and facilitycapacity expansion; (iv) compared with other inexact mixedinteger programming approaches (e.g., ITSMILP by Li and Huang [7]), the GFIP can analyze the inherent interrelationship between the uncertainty of fuzzy parameters (i.e., $\alpha$-cut levels) and capacity expansion options of waste management facilities, and such analysis can help decision makers make tradeoffs between system reliability and system cost. Then, a case study will be provided to demonstrate applicability of the GFIP method to support dynamic analysis for MSW management under uncertainty. The results will be used for generating different decision alternatives under various system conditions and thus for helping identify desired waste management policies.

\section{Methodology}

2.1. Formulation of the Generalized Fuzzy Integer Programming. A GFIP model, with ambiguous coefficients and decision variables expressed as fuzzy sets, can be formulated as follows:

$$
\operatorname{Max} \tilde{f}=\widetilde{c} \times \widetilde{X}
$$

subject to

$$
\begin{gathered}
\tilde{A} \times \widetilde{X} \leq \widetilde{b} \\
\widetilde{X} \geq 0 \\
\tilde{x}_{j}=\text { fuzzy continuous variables, } \quad \tilde{x}_{j} \in \widetilde{X}, \\
j=1,2, \ldots, p \quad(p<n) \\
\tilde{x}_{j}=\text { fuzzy integer variables, } \quad \tilde{x}_{j} \in \widetilde{X}, \\
j=p+1, p+2, \ldots, n,
\end{gathered}
$$

where $\widetilde{c} \in\{\widetilde{R}\}^{1 \times n}, \widetilde{X} \in\{\widetilde{R}\}^{n \times 1}, \widetilde{b} \in\{\widetilde{R}\}^{m \times 1}$, and $\widetilde{A} \in\{\widetilde{R}\}^{m \times n} ; \widetilde{R}$ denotes a set of fuzzy sets; $\widetilde{c}=\left(\widetilde{c}_{1}, \widetilde{c}_{2}, \ldots, \widetilde{c}_{n}\right), \widetilde{X}^{T}=$ $\left(\widetilde{x}_{1}, \tilde{x}_{2}, \ldots, \widetilde{x}_{n}\right), \widetilde{b}^{T}=\left(\widetilde{b}_{1}, \widetilde{b}_{2}, \ldots, \widetilde{b}_{n}\right)$, and $\widetilde{A}=\left(\widetilde{a}_{i j}\right)_{m \times n}$, for all $i \in m, j \in n$. A fuzzy set $(\widetilde{A})$ in $X$ can be defined as $\left\{x, \mu_{\widetilde{A}}(x) \mid x \in X, \mu_{\widetilde{A}}(x): X \rightarrow[0,1]\right\}$, where $\mu_{\widetilde{A}}(x)$ is the membership function or grade of membership [22]. If all elements in $\widetilde{A}$ are integers and $\mu_{\widetilde{A}}(x)$ is a discrete membership function, then $\widetilde{A}$ is a fuzzy integer set [23]. The value of $\mu_{\widetilde{A}}(x)$ varies between 0 and 1 , indicating the possibility of an element $x$ belonging to $\widetilde{A} \cdot \mu_{\widetilde{A}}(x)=1$ means that $x$ definitely belongs to the fuzzy set $(\widetilde{A})$, while $\mu_{\widetilde{A}}(x)=0$ denotes that $x$ does not belong to $\widetilde{A}$. The closer $\mu_{\widetilde{A}}(x)$ is to 1 , the more likely that $x$ belongs to $\widetilde{A}$; conversely, the closer $\mu_{\widetilde{A}}(x)$ is to 0 , the less likely that $x$ belongs to $\widetilde{A}[22,24]$. An $\alpha$-cut of $\widetilde{A}$ can be defined as an ordinary set (denoted as $[\widetilde{A}]_{\alpha}$ ) in which the membership degrees of elements exceed $\alpha .[\widetilde{A}]_{\alpha}$ is usually a continuous or 
discrete fuzzy interval. Consequently, through the concept of $\alpha$-cut, each fuzzy parameter can be characterized as a series of intervals under different $\alpha$-cut levels. Then, interval analysis methods can be applied to process these fuzzy intervals.

\subsection{Solution Method of GFIP Model through Stepwise Interac-} tive Algorithm. If the parameters and variables in model (la)(1e) are triangular fuzzy numbers, several methods can be applied to solve the model, such as the lexicography method proposed by Hosseinzadeh Lotfi et al. [18] and the methods of Fan et al. [12] and Kumar et al. [21]. However, when the parameters of model (1a)-(1e) are expressed through other kinds of fuzzy numbers, the above methods are not applicable. Consequently, in this study, a new method named stepwise interactive algorithm (SIA) will be proposed to solve model (1a)-(1e). This algorithm is based on computational principles related to fuzzy intervals [25-28] (see Appendix section). The detailed proof of the solution algorithm can be found in Fan et al. [20]. The inherent idea of the stepwise interactive algorithm is based on the design of experiment, in which the optimization model would be considered as an experiment with the $\alpha$-cut levels being the inputs and the optimal solutions being the outputs. The detailed procedures of the SIA method include (i) discretizing the membership function grade of fuzzy parameters into a set of $\alpha$-cut levels; (ii) converting the GFIP problem into an inexact mixedinteger linear programming (IMILP) problem under each $\alpha$ cut level; (iii) solving the IMILP problem through an interactive algorithm; and (iv) approximating the membership function for decision variables through statistical regression methods. Compared with the previous methods, SIA can allow uncertainties to be directly communicated into the optimization process. Moreover, it will not lead to complex intermediate submodels and thus lead to a relatively low computational requirement. This is meaningful when the SIA method is applied to solve large-scale management models. Finally, the proposed SIA method can generate solutions expressed as fuzzy sets.

Since the parameters in model (1a)-(1e) are expressed as fuzzy sets, these parameters will be defuzzified before the model is solved. Various defuzzification methods have been proposed to convert fuzzy sets into crisp sets, including $\alpha$ cut, max-membership principle, centroid, weighted average, mean-max membership, center of sums, center of largest, and first of maxima or last of maxima methods. In this study, the $\alpha$-cut would be applied to defuzzify the fuzzy parameters in model (1a)-(1e) due to its popularity and ease of implementation. The concept of $\alpha$-cut is important in reflecting the relationship between fuzzy sets and crisp sets. Each fuzzy set can be uniquely represented by all of its $\alpha$ cuts. As stated by Kreinovich [29], fuzzy data processing is computable for $\alpha$-cuts but, in general, not computable for membership functions. Consequently, the fuzzy parameters and decision variables in model (1a)-(le) are defuzzified through the $\alpha$-cut method instead of their membership functions. Through the $\alpha$-cut method, the fuzzy parameters and decision variables in model (1a)-(1e) will be converted into the related fuzzy intervals. The optimization model with interval parameters can then be transformed into deterministic submodels, which can be solved through ordinary solution methods (e.g., simplex method). Therefore, before solving model (1a)-(1e), a set of $\alpha$-cut levels (i.e., $\alpha_{1}, \alpha_{2}, \ldots, \alpha_{q}$ ) are selected from the unit interval $[0,1]$. Then, for any $\alpha_{i} \in[0,1]$, the associated $\alpha$-cuts for $\widetilde{c}_{j}, \widetilde{x}_{j}, \widetilde{a}_{i j}$, and $\widetilde{b}_{i}$ can be expressed as $\left(\widetilde{c}_{j}\right)_{\alpha_{i}}=\left[\left(c_{j}\right)_{\alpha_{i}}^{-},\left(c_{j}\right)_{\alpha_{i}}^{+}\right],\left(\widetilde{x}_{j}\right)_{\alpha_{i}}=\left[\left(x_{j}\right)_{\alpha_{i}}^{-},\left(x_{j}\right)_{\alpha_{i}}^{+}\right],\left(\widetilde{a}_{i j}\right)_{\alpha_{i}}=$ $\left[\left(a_{i j}\right)_{\alpha_{i}}^{-},\left(a_{i j}\right)_{\alpha_{i}}^{+}\right]$, and $\left(\widetilde{b}_{i}\right)_{\alpha_{i}}=\left[\left(b_{i}\right)_{\alpha_{i}}^{-},\left(b_{i}\right)_{\alpha_{i}}^{+}\right]$.

Rank these $\alpha$-cut levels into an increasing sequence: $\alpha_{(1)}, \alpha_{(2)}, \ldots, \alpha_{(q)}$, where $\alpha_{(1)} \leq \alpha_{(2)} \leq \cdots \leq \alpha_{(q)}$. The minimum $\alpha$-cut level [i.e $\alpha_{(1)}$ ] will be appointed firstly to cut model (1a)-(1e). Then an inexact mixed-integer linear programming (IMILP) model can be formulated as follows:

$$
\operatorname{Max}(f)_{\alpha_{(1)}}^{ \pm}=\sum_{j=1}^{n}\left(c_{j}\right)_{\alpha_{(1)}}^{ \pm} \times\left(x_{j}\right)_{\alpha_{(1)}}^{ \pm}
$$

subject to

$$
\begin{array}{r}
\sum_{j=1}^{n}\left(a_{i j}\right)_{\alpha_{(1)}}^{ \pm} \times\left(x_{j}\right)_{\alpha_{(1)}}^{ \pm} \leq\left(b_{i}\right)_{\alpha_{(1)}}^{ \pm} \quad i=1,2, \ldots, m \quad(2 \mathrm{~b}) \\
\left(x_{j}\right)_{\alpha_{(1)}}^{ \pm} \geq 0 \quad j=1,2, \ldots, m \quad(2 \mathrm{c}) \\
\left(x_{j}\right)_{\alpha_{(1)}}^{ \pm} \text {interval continuous variables, }\left(x_{j}\right)_{\alpha_{(1)}}^{ \pm} \in(X)_{\alpha_{(1)}}^{ \pm}, \\
=1,2, \ldots, p \quad(p<n), \\
(2 \mathrm{~d})
\end{array}
$$

where $(f)_{\alpha_{(1)}}^{ \pm},\left(c_{j}\right)_{\left.\alpha_{(1)}\right)}^{ \pm},\left(x_{j}\right)_{\alpha_{(1)}}^{ \pm},\left(a_{i j}\right)_{\alpha_{(1)}}^{ \pm}$, and $\left(b_{i}\right)_{\alpha_{(1)}}^{ \pm}$are fuzzy intervals under $\alpha_{(1)} \cdot\left(c_{j}\right)_{\alpha_{(1)}}^{ \pm}=\left[\left(c_{j}\right)_{\alpha_{(1)}}^{-},\left(c_{j}\right)_{\alpha_{(1)}}^{+}\right] ;\left(x_{j}\right)_{\alpha_{(1)}}^{ \pm}=$ $\left[\left(x_{j}\right)_{\alpha_{(1)}}^{-},\left(x_{j}\right)_{\alpha_{(1)}}^{+}\right] ;\left(a_{i j}\right)_{\alpha_{(1)}}^{ \pm}=\left[\left(a_{i j}\right)_{\alpha_{(1)}}^{-},\left(a_{i j}\right)_{\alpha_{(1)}}^{+}\right] ;$and $\left(b_{i}\right)_{\alpha_{(1)}}^{ \pm}=$ $\left[\left(b_{i}\right)_{\alpha_{(1)}}^{-},\left(b_{i}\right)_{\alpha_{(1)}}^{+}\right]$. Fuzzy intervals under other $\alpha$-cut levels also have similar expressions. Furthermore, an interval number $\left(a^{ \pm}\right)$can be defined as $a^{ \pm}=\left[a^{-}, a^{+}\right]=\left\{t \mid a^{-} \leq t \leq a^{+}\right\}$.

Model (2a)-(2e) shows the formulation of interval mixedinteger linear programming (IMILP) method with all parameters expressed as interval numbers. The IMILP model was developed through introducing the concept of interval analysis into a mixed-integer linear programming framework [3]. It allowed uncertainties to be directly communicated into the optimization processes and resulting solutions and did not lead to complicated intermediate models [3].

Since model (2a)-(2e) is an inexact optimization model with all parameters expressed as intervals, it can be solved through the interactive algorithm proposed by Huang et al. [3]. Assume that the former $k_{1}$ coefficients of model (2a)(2e) are positive and the latter $k_{2}$ coefficients are negative $\left(k_{1}+k_{2}=n\right)$. Then model $(2 \mathrm{a})-(2 \mathrm{e})$ can be converted into 
two submodels. In detail, the first submodel corresponding to $(f)_{\alpha_{(1)}}^{+}$can be formulated as:

$\operatorname{Max}(f)_{\alpha_{(1)}}^{+}=\sum_{j=1}^{k_{1}}\left(c_{j}\right)_{\alpha_{(1)}}^{+}\left(x_{j}\right)_{\alpha_{(1)}}^{+}+\sum_{j=k_{1}+1}^{n}\left(c_{j}\right)_{\alpha_{(1)}}^{+}\left(x_{j}\right)_{\alpha_{(1)}}^{-}$

subject to

$$
\begin{gathered}
\sum_{j=1}^{k} \operatorname{Sign}\left(\left(a_{i j}\right)_{\alpha_{(1)}}^{ \pm}\right)\left|\left(a_{i j}\right)_{\alpha_{(1)}}\right|^{-}\left(x_{j}\right)_{\alpha_{(1)}}^{+} \\
+\sum_{j=k+1}^{n} \operatorname{Sign}\left(\left(a_{i j}\right)_{\alpha_{(1)}}^{ \pm}\right)\left|\left(a_{i j}\right)_{\alpha_{(1)}}\right|^{+}\left(x_{j}\right)_{\alpha_{(1)}}^{-} \\
\leq\left(b_{i}\right)_{\alpha_{(1)}}^{+}, \quad \forall i \\
\left(x_{j}\right)_{\alpha_{(1)}}^{ \pm}=\text {interval continuous variables, } \\
j=1,2, \ldots, p_{1} ; k_{1}+1, k_{1}+2, \ldots, k_{1}+p_{2} ; \\
\left(p_{1} \leq k_{1}, p_{2} \leq k_{2}, k_{1}+k_{2}=n\right) \\
\left(x_{j}\right)_{\alpha_{(1)}}^{ \pm}=\text {interval discrete variables, } \\
j=p_{1}+1, p_{1}+2, \ldots, k_{1} ; \\
k_{1}+p_{2}+1, k_{1}+p_{2}+2, \ldots, n \\
\left(x_{j}\right)_{\alpha_{(1)}}^{ \pm} \geq 0, \quad \forall j .
\end{gathered}
$$

Solutions of $\left(x_{\text {jopt }}\right)_{\alpha_{(1)}}^{+}\left(j=1,2, \ldots, k_{1}\right)$ and $\left(x_{\text {jopt }}\right)_{\alpha_{(1)}}^{-}(j=$ $\left.k_{1}+1, k_{1}+2, \ldots, n\right)$ can be obtained from submodel (3a)-(3e). Then the second submodel corresponding to $(f)_{\alpha_{(1)}}^{-}$can be formulated based on solutions from the first submodel, which can be expressed as follows:

$$
\operatorname{Max}(f)_{\alpha_{(1)}}^{-}=\sum_{j=1}^{k}\left(c_{j}\right)_{\alpha_{(1)}}^{-}\left(x_{j}\right)_{\alpha_{(1)}}^{-}+\sum_{j=k+1}^{n}\left(c_{j}\right)_{\alpha_{(1)}}^{-}\left(x_{j}\right)_{\alpha_{(1)}}^{+}
$$

subject to

$$
\begin{aligned}
& \sum_{j=1}^{k} \operatorname{Sign}\left(\left(a_{i j}\right)_{\alpha_{(1)}}^{ \pm}\right)\left|\left(a_{i j}\right)_{\alpha_{(1)}}\right|^{+}\left(x_{j}\right)_{\alpha_{(1)}}^{-} \\
& \quad+\sum_{j=k+1}^{n} \operatorname{Sign}\left(\left(a_{i j}\right)_{\alpha_{(1)}}^{ \pm}\right)\left|\left(a_{i j}\right)_{\alpha_{(1)}}\right|^{-}\left(x_{j}\right)_{\alpha_{(1)}}^{+} \\
& \leq\left(b_{i}\right)_{\alpha_{(1)}}^{-}, \quad \text { for all } i
\end{aligned}
$$

$$
\begin{array}{r}
\left(x_{j}\right)_{\alpha_{(1)}}^{ \pm}=\text {interval continuous variables, } \\
j=1,2, \ldots, p_{1} ; k_{1}+1, k_{1}+2, \ldots, k_{1}+p_{2}, \\
\left(p_{1} \leq k_{1}, p_{2} \leq k_{2}, k_{1}+k_{2}=n\right)
\end{array}
$$

$\left(x_{j}\right)_{\alpha_{(1)}}^{ \pm}=$interval discrete variables,

$$
j=p_{1}+1, p_{1}+2, \ldots, k_{1}
$$

$$
\begin{gathered}
k_{1}+p_{2}+1, k_{1}+p_{2}+2, \ldots, n \\
\left(x_{j}\right)_{\alpha_{(1)}}^{-} \leq\left(x_{\text {jopt }}\right)_{\alpha_{(1)}}^{+}, \quad j=1,2, \ldots, k_{1} \\
\left(x_{j}\right)_{\alpha_{(1)}}^{+} \geq\left(x_{j \mathrm{opt}}\right)_{\alpha_{(1)}}^{-}, \quad j=k_{1}+1, k_{1}+2, \ldots, n \\
\left(x_{j}\right)_{\alpha_{(1)}}^{ \pm} \geq 0, \quad \forall j .
\end{gathered}
$$

Hence, solutions of $\left(x_{j}\right)_{\alpha_{(1)}}^{-}\left(j=1,2, \ldots, k_{1}\right)$ and $\left(x_{j}\right)_{\alpha_{(1)}}^{+}\left(j=k_{1}+1, k_{1}+2, \ldots, n\right)$ can be obtained from submodel $(4 \mathrm{a})-(4 \mathrm{~g})$. Therefore, the final solutions for model (2a)-(2e) can be generated, which are presented as follows:

$$
\begin{aligned}
& \left(x_{\text {jopt }}\right)_{\alpha_{(1)}}^{ \pm}=\left[\left(x_{\text {jopt }}\right)_{\alpha_{(1)}}^{-}\left(x_{\text {jopt }}\right)_{\alpha_{(1)}}^{+}\right] \\
& \left(f_{\text {opt }}\right)_{\alpha_{(1)}}^{ \pm}=\left[\left(f_{\text {opt }}\right)_{\alpha_{(1)}}^{-}\left(f_{\text {opt }}\right)_{\alpha_{(1)}}^{+}\right] \text {. }
\end{aligned}
$$

Formulas $((3 a)-(3 e))$ to $((5 a)-(5 b))$ show the detailed solution process of an IMILP model through the interactive algorithm (also named two-step method). Based on the interactive algorithm, the original IMILP model is firstly reformulated into two submodels corresponding, respectively, to its upper and lower bounds of objective function; the two submodels are then solved separately one after another [30]. The sequence to solve two submodels is subject to the nature of objective function (max or min). For a maximized problem [i.e., model (2a)-(2e)], the submodel corresponding to the upper bound of the objective function is solved first, followed by solving the submodel corresponding to the lower bound of the objective function; besides, the optimal solutions from the first submodel should be used as constraints for the second submodel [30].

Based on solutions of model (2a)-(2e), we will select $\alpha_{(2)}$ to $\alpha_{(q)}$ in sequence and then formulate corresponding IMILP models as follows:

$$
\operatorname{Max}(f)_{\alpha_{(l)}}^{ \pm}=\sum_{j=1}^{n}\left(c_{j}\right)_{\alpha_{(l)}}^{ \pm} \times\left(x_{j}\right)_{\alpha_{(l)}}^{ \pm}
$$


subject to

$$
\begin{gathered}
\sum_{j=1}^{n}\left(a_{i j}\right)_{\alpha_{(l)}}^{ \pm} \times\left(x_{j}\right)_{\alpha_{(l)}}^{ \pm} \leq\left(b_{i}\right)_{\alpha_{(l)}}^{ \pm}, \quad \text { for } i=1,2, \ldots, m \\
\left(x_{j}\right)_{\alpha_{(l)}}^{ \pm}=\text {interval continuous variables, } \\
j=1,2, \ldots, p_{1} ; k_{1}+1, k_{1}+2, \ldots, k_{1}+p_{2}, \\
\left(p_{1} \leq k_{1}, p_{2} \leq k_{2}, k_{1}+k_{2}=n\right) \\
\left(x_{j}\right)_{\alpha_{(l)}}^{ \pm}=\text {interval discrete variables, } \\
j=p_{1}+1, p_{1}+2, \ldots, k_{1} ; \\
k_{1}+p_{2}+1, k_{1}+p_{2}+2, \ldots, n \\
\left(x_{j}\right)_{\alpha_{(l)}}^{ \pm} \subseteq\left(x_{j \mathrm{opt}}\right)_{\alpha_{(l-1)}}^{ \pm} \\
\left(x_{j}\right)_{\alpha_{(1)}}^{ \pm} \geq 0, \quad \forall j,
\end{gathered}
$$

where $\alpha_{(l)} \in\left\{\alpha_{(2)}, \ldots, \alpha_{(q)}\right\}$ and $\left(x_{j \text { opt }}\right)_{\alpha_{(l-1)}}^{ \pm}$are the optimal solutions obtained from the IMILP model under $\alpha_{(l-1)}$. Formula (6e) is proposed to reflect the property of the fuzzy number that $\left(x_{j}\right)_{\alpha_{1}}^{ \pm} \supseteq\left(x_{j}\right)_{\alpha_{2}}^{ \pm}$holds when $\alpha_{1}, \alpha_{2} \in[0,1]$ and $\alpha_{1} \leq \alpha_{2}$.

Based on the interactive algorithm, model (6a)-(6f) will be converted into two submodels as follows.

Submodel 1:

$\operatorname{Max}(f)_{\alpha_{(l)}}^{+}=\sum_{j=1}^{k_{1}}\left(c_{j}\right)_{\alpha_{(l)}}^{+}\left(x_{j}\right)_{\alpha_{(l)}}^{+}+\sum_{j=k_{1}+1}^{n}\left(c_{j}\right)_{\alpha_{(l)}}^{+}\left(x_{j}\right)_{\alpha_{(l)}}^{-}$

subject to

$$
\begin{aligned}
& \sum_{j=1}^{k} \operatorname{Sign}\left(\left(a_{i j}\right)_{\alpha_{(l)}}^{ \pm}\right)\left|\left(a_{i j}\right)_{\alpha_{(l)}}\right|^{-}\left(x_{j}\right)_{\alpha_{(l)}}^{+} \\
& \quad+\sum_{j=k+1}^{n} \operatorname{Sign}\left(\left(a_{i j}\right)_{\alpha_{(l)}}^{ \pm}\right)\left|\left(a_{i j}\right)_{\alpha_{(l)}}\right|^{+}\left(x_{j}\right)_{\alpha_{(l)}}^{-} \\
& \leq\left(b_{i}\right)_{\alpha_{(l)}}^{+}, \quad \forall i \\
& \left(x_{j}\right)_{\alpha_{(l)}}^{ \pm}=\text {interval continuous variables, }
\end{aligned}
$$

$$
\begin{array}{r}
j=1,2, \ldots, p_{1} ; k_{1}+1, k_{1}+2, \ldots, k_{1}+p_{2}, \\
\left(p_{1} \leq k_{1}, p_{2} \leq k_{2}, k_{1}+k_{2}=n\right)
\end{array}
$$$$
\left(x_{j}\right)_{\alpha_{(l)}}^{ \pm}=\text {interval discrete variables, }
$$$$
j=p_{1}+1, p_{1}+2, \ldots, k_{1} ;
$$$$
k_{1}+p_{2}+1, k_{1}+p_{2}+2, \ldots, n
$$$$
\left(x_{j}\right)_{\alpha_{(l)}}^{+} \leq\left(x_{j \mathrm{opt}}\right)_{\alpha_{(l-1)}}^{+}, \quad j=1,2, \ldots, k_{1}
$$$$
\left(x_{j}\right)_{\alpha_{(l)}}^{-} \geq\left(x_{j \mathrm{opt}}\right)_{\alpha_{(l-1)}}^{-}, \quad j=k_{1}+1, k_{1}+2, \ldots, n
$$

$$
\left(x_{j}\right)_{\alpha_{(l)}}^{ \pm} \geq 0, \quad \forall j .
$$

Submodel 2:

$$
\operatorname{Max}(f)_{\alpha_{(l)}}^{-}=\sum_{j=1}^{k}\left(c_{j}\right)_{\alpha_{(l)}}^{-}\left(x_{j}\right)_{\alpha_{(l)}}^{-}+\sum_{j=k+1}^{n}\left(c_{j}\right)_{\alpha_{(l)}}^{-}\left(x_{j}\right)_{\alpha_{(l)}}^{+}
$$

subject to

$$
\begin{gathered}
\sum_{j=1}^{k} \operatorname{Sign}\left(\left(a_{i j}\right)_{\alpha_{(l)}}^{ \pm}\right)\left|\left(a_{i j}\right)_{\alpha_{(l)}}\right|^{+}\left(x_{j}\right)_{\alpha_{(l)}}^{-} \\
+\sum_{j=k+1}^{n} \operatorname{Sign}\left(\left(a_{i j}\right)_{\alpha_{(l)}}^{ \pm}\right)\left|\left(a_{i j}\right)_{\alpha_{(l)}}\right|^{-}\left(x_{j}\right)_{\alpha_{(l)}}^{+} \\
\leq\left(b_{i}\right)_{\alpha_{(l)}}^{-}, \quad \forall i \\
\left(x_{j}\right)_{\alpha_{(l)}^{ \pm}}^{ \pm}=\text {interval continuous variables, } \\
j=1,2, \ldots, p_{1} ; k_{1}+1, k_{1}+2, \ldots, k_{1}+p_{2}, \\
\left(p_{1} \leq k_{1}, \quad p_{2} \leq k_{2}, k_{1}+k_{2}=n\right) \\
\left(x_{j}\right)_{\alpha_{(l)}}^{ \pm}=\text {interval discrete variables, } \\
j=p_{1}+1, p_{1}+2, \ldots, k_{1} ; \\
k_{1}+p_{2}+1, k_{1}+p_{2}+2, \ldots, n \\
\left(x_{j}\right)_{\alpha_{(l)}}^{-} \leq\left(x_{j o p t}\right)_{\alpha_{(l)}}^{+}, \quad j=1,2, \ldots, k_{1} \\
\geq\left(x_{j o p t}\right)_{\alpha_{(l)}}^{-}, \quad j=k_{1}+1, k_{1}+2, \ldots, n \\
\left(x_{j}\right)_{\alpha_{(l)}}^{+} \geq\left(x_{j o p t}\right)_{\alpha_{(l-1)}}^{-}, \quad j=1,2, \ldots, k_{1} \\
\leq\left(x_{j o p t}\right)_{\alpha_{(l-1)}}^{+} \quad j=k_{1}+1, k_{1}+2, \ldots, n \\
\left(x_{j}\right)_{\alpha_{(l)}}^{ \pm} \geq 0, \quad \forall j .
\end{gathered}
$$


From submodels $((7 a)-(7 g))$ and $((8 a)-(8 i))$, we can obtain the final solutions for model $(6 a)-(6 f)$ under $\alpha_{(l)}(l=$ $2,3, \ldots, q)$ as follows:

$$
\begin{aligned}
\left(x_{\text {jopt }}\right)_{\alpha_{(l)}}^{ \pm} & =\left[\begin{array}{ll}
\left(x_{\text {jopt }}\right)_{\alpha_{(l)}}^{-} & \left(x_{\text {jopt }}\right)_{\alpha_{(l)}}^{+}
\end{array}\right] \\
\left(f_{\text {opt }}\right)_{\alpha_{(l)}}^{ \pm} & =\left[\begin{array}{ll}
\left(f_{\text {opt }}\right)_{\alpha_{(l)}}^{-} & \left(f_{\text {opt }}\right)_{\alpha_{(l)}}^{+}
\end{array}\right] .
\end{aligned}
$$

Based on formulas $((2 a)-(2 e))-((9 a)-(9 b))$, we can obtain a series of fuzzy interval solutions for model (1a)-(1e) under different $\alpha$-cut levels. Then we can approximate the membership function for continuous decision variables by statistical regression methods. In this procedure, the GFIP model is supposed to be an experiment, with $\alpha$-cut levels being its inputs (i.e., independent variables) and the lower and upper bounds of decision variables being its outputs (i.e., dependent variables). Take $\left(x_{\text {jopt }}\right)_{\alpha}^{-}$as an example, we can obtain a regression function between $\left(x_{\text {jopt }}\right)_{\alpha}^{-}$and $\alpha$ based on the fuzzy interval solutions. Such a regression function will be considered as the inverse function of the left shape function for $\tilde{x}_{j}$, denoted as $L^{-1}(x)$; then we can acquire the left shape function for $\tilde{x}_{j}$, expressed as $L(x)$. In the same way, we can obtain the right shape function for $\tilde{x}_{j}$, expressed as $R(x)$.

\section{Case Study}

A hypothetical municipal solid waste (MSW) management problem is used to illustrate the applicability of GFIP approach. The studied system includes three municipal cities. A planning horizon of 15 years is divided into three periods, with each one having a time interval of 5 years. Two types of facilities can be available for waste treatment/disposal. A landfill is considered in the proposed case due to its crucial role for MSW disposal in both developed and developing countries. For example, more than 54 percent of MSW was landfilled in the United States during 2009 [31], while 89.3 percent of the generated MSW (74.04 million tonnes) was landfilled in China in 2002 [32]. The landfill is typically characterized as an overall capacity limit. Also, a waste-toenergy (WTE) facility, which can effectively minimize land depletion caused by landfilling, is employed to serve wastedisposal needs. It is characterized as a daily capacity limit.

In fact, a MSW management system involves several processes with socioeconomic and environmental implications, such as waste generation, transportation treatment, and disposal [33]. Extensive uncertainties usually exist in these processes due to impacts of the economic development, population growth, and human activities. Moreover, probabilistic methods are not applicable to quantify these uncertainties when data are insufficient. Consequently, adoption of fuzzy set theory would be a potential alternative, especially when uncertainties can be consciously assumed by decision makers or experts. Furthermore, uncertain inputs in the MSW management system would lead to variations in the resulting solutions. Therefore, the GFIP method will be desired to reflect uncertain and dynamic complexities in the MSW management system and generate solutions expressed as fuzzy sets.
Table 1 shows related waste generation levels and cost coefficients, including waste generation rates in three cities, operation costs of two facilities, and transportation costs for shipping waste flows. These parameters are estimated as triangular fuzzy numbers with known most possible values, as well as left and right spreads. Table 2 presents capacity expansion options and related costs for waste disposal facilities. The total capacity of landfill is $\left(1.8 \times 10^{6}, 0.2 \times 10^{5}, 0.3 \times 10^{5}\right)$ tonne, which means the most possible capacity of landfill is $1.8 \times 10^{6}$ tonne, and the lower and upper bound is $1.78 \times 10^{6}$ and $1.83 \times 10^{6}$ tonne, respectively. The daily capacity of WTE facility is $(390,20$, and 20$)$ tonne/day, which means the most possible capacity and lower and upper bound is 390, 370, and 410 tonne/day, respectively. The WTE facility will generate residues of about $30 \%$ (10\% as its left and right spread) of the incoming waste stream. The revenue from the WTE facility is approximately $\$ 20 /$ tonne, with its left and right spreads being $\$ 2 /$ tonne. In this study, all parameters are assumed to be triangular fuzzy numbers. The triangular fuzzy numbers are considered in this study because (i) the triangular form is the simplest type of fuzzy numbers, (ii) many other types of fuzzy numbers can be estimated through the triangular fuzzy numbers, and (iii) triangular membership function can provide the most important information for a fuzzy set: lower-bound value, upper-bound value, and the most possible value [34]. Also, other kinds of fuzzy numbers can be treated through the proposed GFIP approach if their membership functions are known.

The problem under consideration is how to effectively allocate waste flows and choose appropriate capacity expansion options of waste-disposal facilities under a number of environmental, economic, and treatment/disposal constraints in order to minimize the overall system cost. A GFIP model can thus be formulated to solve this problem.

In this study, decision variable $\tilde{x}_{i j k}$ represents the amount of waste flow from city $j$ to waste-treatment facility $i$ in period $k$. The objective is to minimize the system cost through effectively allocating waste flows from three cities to two disposal facilities and choosing appropriate waste-disposalfacility options for excessive waste-disposal requirements. The constraints involve relationships between decision variables and waste generation/management conditions. Thus, a GFIP model can be formulated as follows:

$$
\begin{gathered}
\operatorname{Min} \tilde{f}=1825 \sum_{j=1}^{3} \sum_{k=1}^{3}\left\{\sum_{i=1}^{2} \widetilde{x}_{i j k}\left(\widetilde{\mathrm{TR}}_{i j k}+\widetilde{\mathrm{OP}}_{i k}\right)+\widetilde{x}_{2 j k}\right. \\
\times\left[\widetilde{\mathrm{FE}}\left(\widetilde{\mathrm{FT}}_{k}+\widetilde{\mathrm{OP}}_{1 k}\right)\right. \\
\left.\left.-\widetilde{\mathrm{RE}}_{k}\right]\right\} \\
+\sum_{k=1}^{3} \widetilde{\mathrm{FLC}}_{k} \widetilde{Y}_{k}+\sum_{l=1}^{3} \sum_{k=1}^{3} \widetilde{\mathrm{FTC}}_{l k} \widetilde{Z}_{l k}
\end{gathered}
$$

subject to 
TABLE 1: Waste generation levels and cost coefficients.

\begin{tabular}{|c|c|c|c|}
\hline & \multicolumn{3}{|c|}{ Time period } \\
\hline & $k=1$ & $k=2$ & $k=3$ \\
\hline \multicolumn{4}{|c|}{ Waste generation $\widetilde{\mathrm{WG}}_{j k}$ (t/day) } \\
\hline City 1 & $(225,25,25)$ & $(250,25,25)$ & $(275,25,25)$ \\
\hline City 2 & $(375,25,25)$ & $(400,25,25)$ & $(425,25,25)$ \\
\hline City 3 & $(300,25,25)$ & $(325,25,25)$ & $(350,25,25)$ \\
\hline \multicolumn{4}{|c|}{$\begin{array}{l}\text { Cost of transportation to landfill } \widetilde{\mathrm{TR}}_{1 j k} \\
(\$ / \mathrm{t})\end{array}$} \\
\hline City 1 & $(14.1,2,2)$ & $(15.5,2.2,2.2)$ & $(17,2.4,2.5)$ \\
\hline City 2 & $(12,1.5,2)$ & $(13,1.9,1.9)$ & $(15,2.2,1.9)$ \\
\hline City 3 & $(15.1,2.4,1.9)$ & $(16.5,2.5,2.2)$ & $(18,2.6,2.6)$ \\
\hline \multicolumn{4}{|c|}{$\begin{array}{l}\text { Cost of transportation to landfill } \widetilde{\mathrm{FT}}_{k} \\
(\$ / \mathrm{t})\end{array}$} \\
\hline Waste-to-energy facility & $(10,1,1)$ & $(12,1,1)$ & $(14,1,1)$ \\
\hline \multicolumn{4}{|c|}{$\begin{array}{l}\text { Cost of transportation to } \\
\text { waste-to-energy facility } \widetilde{\mathrm{TR}}_{2 j k}(\$ / \mathrm{t})\end{array}$} \\
\hline City 1 & $(11.2,1.6,1.6)$ & $(12.3,1.5,1.5)$ & $(13.5,1.8,2)$ \\
\hline City 2 & $(11.8,1.7,1.6)$ & $(12.9,1.8,1.8)$ & $(14.2,2,2)$ \\
\hline City 3 & $(10.2,1.4,1.5)$ & $(11.3,1.4,1.5)$ & $(12.6,1.7,1.7)$ \\
\hline \multicolumn{4}{|l|}{ Operation costs $\widetilde{\mathrm{OP}}_{i k}(\$ / \mathrm{t})$} \\
\hline Landfill & $(50,8,7)$ & $(58,10,10)$ & $(68,15,15)$ \\
\hline Waste-to-energy facility & $(60,10,10)$ & $(68,10,10)$ & $(75,10,10)$ \\
\hline
\end{tabular}

TABLE 2: Capacity expansion options and costs for landfill and WTE facilities.

\begin{tabular}{|c|c|c|c|}
\hline \multirow{2}{*}{ Data } & \multicolumn{3}{|c|}{ Time period } \\
\hline & $k=1$ & $k=2$ & $k=3$ \\
\hline \multicolumn{4}{|c|}{$\begin{array}{l}\text { Capacity expansion options for WTE } \\
\text { facility (tonne/day) }\end{array}$} \\
\hline$\widetilde{\Delta \mathrm{TC}}_{1}($ option 1$)$ & 150 & 150 & 150 \\
\hline$\widetilde{\Delta \mathrm{TC}}_{2}($ option 1$)$ & 200 & 200 & 200 \\
\hline$\widetilde{\Delta \mathrm{TC}}_{3}($ option 1$)$ & 250 & 250 & 250 \\
\hline \multicolumn{4}{|c|}{$\begin{array}{l}\text { Capacity expansion options for the } \\
\text { landfill facility }\left(10^{6} \text { tonne }\right)\end{array}$} \\
\hline$\widetilde{\Delta \mathrm{TC}}$ & $(0.31,0.01,0.015)$ & $(0.31,0.01,0.015)$ & $(0.31,0.01,0.015)$ \\
\hline \multicolumn{4}{|c|}{ Capital cost for WTE expansion $\left(\$ 10^{6}\right)$} \\
\hline$\widetilde{\mathrm{FTC}}_{1 k}$ (option 1) & 10.5 & 8.3 & 6.5 \\
\hline$\widetilde{\mathrm{FTC}}_{2 k}$ (option 1$)$ & 15.2 & 11.9 & 9.3 \\
\hline$\widetilde{\mathrm{FTC}}_{3 k}$ (option 1) & 19.8 & 15.5 & 12.2 \\
\hline \multicolumn{4}{|c|}{$\begin{array}{l}\text { Capital cost for landfill expansion } \\
\left(\$ 10^{6}\right)\end{array}$} \\
\hline$\widetilde{\mathrm{FLC}}_{k}$ & $(14,1,1)$ & $(14,1,1)$ & $(14,1,1)$ \\
\hline
\end{tabular}

(1) Landfill capacity constraint:

$$
\begin{array}{r}
1825 \sum_{j=1}^{3} \sum_{k=1}^{k^{\prime}}\left(\tilde{x}_{1 j k}+\tilde{x}_{2 j k} \widetilde{\mathrm{FE}}\right) \leq \widetilde{\mathrm{TL}} \\
+\widetilde{\Delta \mathrm{TC}} \sum_{k=1}^{k^{\prime}} \widetilde{Y}_{k} \quad k^{\prime}=1,2,3 .
\end{array}
$$

(2) WTE facility-capacity constraints:

$$
\sum_{j=1}^{3} \tilde{x}_{2 j k^{\prime}} \leq \widetilde{\mathrm{TE}}+\sum_{l=1}^{3} \sum_{k=1}^{k^{\prime}} \widetilde{\Delta \mathrm{TC}_{\mathrm{l}}} \widetilde{Z}_{l k} \quad k^{\prime}=1,2,3 .
$$

(3) Waste disposal demand constraints:

$$
\sum_{i=1}^{2} \tilde{x}_{i j k} \geq \widetilde{\mathrm{WG}}_{j k} \quad \forall j, k .
$$


(4) Nonnegativity constraints:

$$
\tilde{x}_{i j k} \geq 0 \quad \forall i, j, k .
$$

(5) Nonnegativity and binary constraints:

$$
\begin{aligned}
& \tilde{Y}_{k}=\left\{\begin{array}{l}
\leq 1 \\
\geq 0 \\
=\text { integer } \quad \forall k .
\end{array}\right. \\
& \widetilde{Z}_{l k}=\left\{\begin{array}{l}
\leq 1 \\
\geq 0 \\
=\text { integer } \quad \forall l, k .
\end{array}\right.
\end{aligned}
$$

(6) Landfill expansion constraint:

$$
\sum_{k=1}^{3} \tilde{Y}_{k} \leq 1
$$

(7) WTE facility expansion constraints:

$$
\sum_{l=1}^{3} \widetilde{Z}_{l k} \leq 1 \quad \forall k
$$

where $\widetilde{\mathrm{FE}}$ is the residue flow from WTE to landfill (\% of incoming mass to WTE facility); $\widetilde{\mathrm{FLC}}_{k}$ is the capital cost of landfill expansion in period $k(\$) ; \widetilde{\mathrm{FT}}_{k}$ is the transportation cost of waste flow from WTE to landfill in period $k$ ( $\$ /$ tonne); $\widetilde{\mathrm{FTC}}_{l k}$ is the capital cost of expanding WTE by option $l$ in period $k(\$) ; \widetilde{\mathrm{OP}}_{i k}$ is the operating cost of facility $i$ in period $k$ (\$/tonne); $\widetilde{\mathrm{RE}}_{k}$ is the revenue from WTE in period $k$ (\$/tonne); $\widetilde{\mathrm{TE}}$ is the maximum capacity of WTE facility (tonne/day); $\widetilde{\mathrm{TL}}$ is the capacity of landfill (tonne); $\widetilde{\mathrm{TR}}_{i j k}$ is the transportation cost for waste flow from city $j$ to facility $i$ during period $k$ (\$/tonne); $\widetilde{\mathrm{WG}}_{j k}$ is the waste generation rate in city $j$ during period $k$ (tonne/day); $\widetilde{\Delta \mathrm{TC}}$ is the total amount of expansion capacity for landfill (tonne); $\widetilde{\Delta \mathrm{TC}}_{l}$ is the amount of the $l$ th type of expansion capacity for WTE (tone/day); $\tilde{x}_{i j k}$ is the waste-flow rate from city $j$ to facility $i$ in period $k$ (tonne/day), $i=1,2 ; j=1,2,3 ; k=1,2,3 ; \widetilde{Y}_{k}$ is the binary decision variable for landfill expansion at the start of period $k ; \widetilde{Z}_{l k}$ is the binary decision variable for WTE facility with expansion option $l$ at the start period of $k$; $i$ is the index for facility ( $i=1$ for landfill and $i=2$ for WTE facility); $j$ is the index for three cities $(j=1,2,3) ; k$ is the index for time periods $(k=1,2,3)$.

In model (10a)-(10i), the objective (i.e., formula (10a)) is to minimize the total cost of facility expansion and wasteflow disposal over the entire planning horizon, which will cover expenses of handling waste flows, charges of expanding facilities, and revenues from the WTE facility. Constraint (10b) specifies that the total amount of waste allocated to the landfill must not exceed its existing and expanded capacities. In this study, one year is assumed to have 365 days, and there are 5 years in each period. Consequently, the coefficient in constraint $(10 b)$ would be 1825 (i.e., $365 \times 5$ ). Constraint (10c) means that the actual daily waste flows shipped to the WTE facility should not exceed its existing and expanded capacities. Constraint (10d) indicates that, for each city in each period, the waste flows transported to the landfill and WTE must be not less than its waste-disposal demand in this period. This constraint also assumes that all solid wastes have to be shipped to a disposal site within a certain period after its generation, and no mass loss is incurred in the transportation process. Constraints (10f) (10i) define the binary variables related to capacity expansion decisions; constraint (10h) denotes that the landfill can only be expanded once within the entire planning horizon, and constraint (10i) means that the WTE can be expanded once in each period.

Figure 1 shows the schematic of the GFIP model for MSW management. Obviously, the GFIP model is an integration of generalized fuzzy linear programming (GFLP) and mixedinteger linear programming (MILP) methods. Each method has a unique contribution in enhancing the capability of GFIP in dealing with uncertainties and dynamic features in solid waste management. For example, fuzzy parameters can be handled by GFLP, and waste management facility expansion can be addressed by MILP. Besides, a stepwise interactive algorithm (SIA) is proposed for solving the proposed GFIP model, which can permit uncertainty to be directly communicated into the optimization process and resulting solutions. Through SIA, the developed GFIP model will firstly be converted into several IMILP submodels and then be further transformed into mixed-integer linear programming (MILP) submodels. Consequently, the computational complexity of the GFIP would be reasonable. For example, if $n \alpha$-cut levels are identified in solving the GFIP model, $n$ IMILP submodels will be firstly generated. According to interactive algorithm, each IMILP submodel can be further converted into two MILP submodels; thus, the GFIP model will finally result in $2 n$ MILP submodels with deterministic parameters.

\section{Result Analysis}

In this study, a GFIP model is developed for supporting decision making in MSW management. A stepwise interactive algorithm (SIA) is proposed to solve the GFIP model. Based on SIA, six $\alpha$-cut levels (i.e., 0, 0.3, 0.5, 0.7, 0.85, and 1) would be considered. Under each $\alpha$-cut level, the fuzzy parameters presented in Tables 1 and 2 would be converted into corresponding fuzzy intervals, and model (10a)-(10i) would also be transformed into an inexact mixedinteger linear programming (IMILP) model. Tables 3 to 5 present waste-flow diversion schemes, capacity expansion options, and related system costs obtained through IMILP models under the selected $\alpha$-cut levels. The results indicate that waste-flow patterns and capacity expansion options would vary due to temporal and spatial variations in waste generation rates and waste management conditions.

In period 1, waste from city 1 would be initially shipped to WTE, with a flow amount of 195 tonne/day. For city 1, the WTE facility would be the first choice to serve its waste disposal requirement. Then the remaining waste would be 


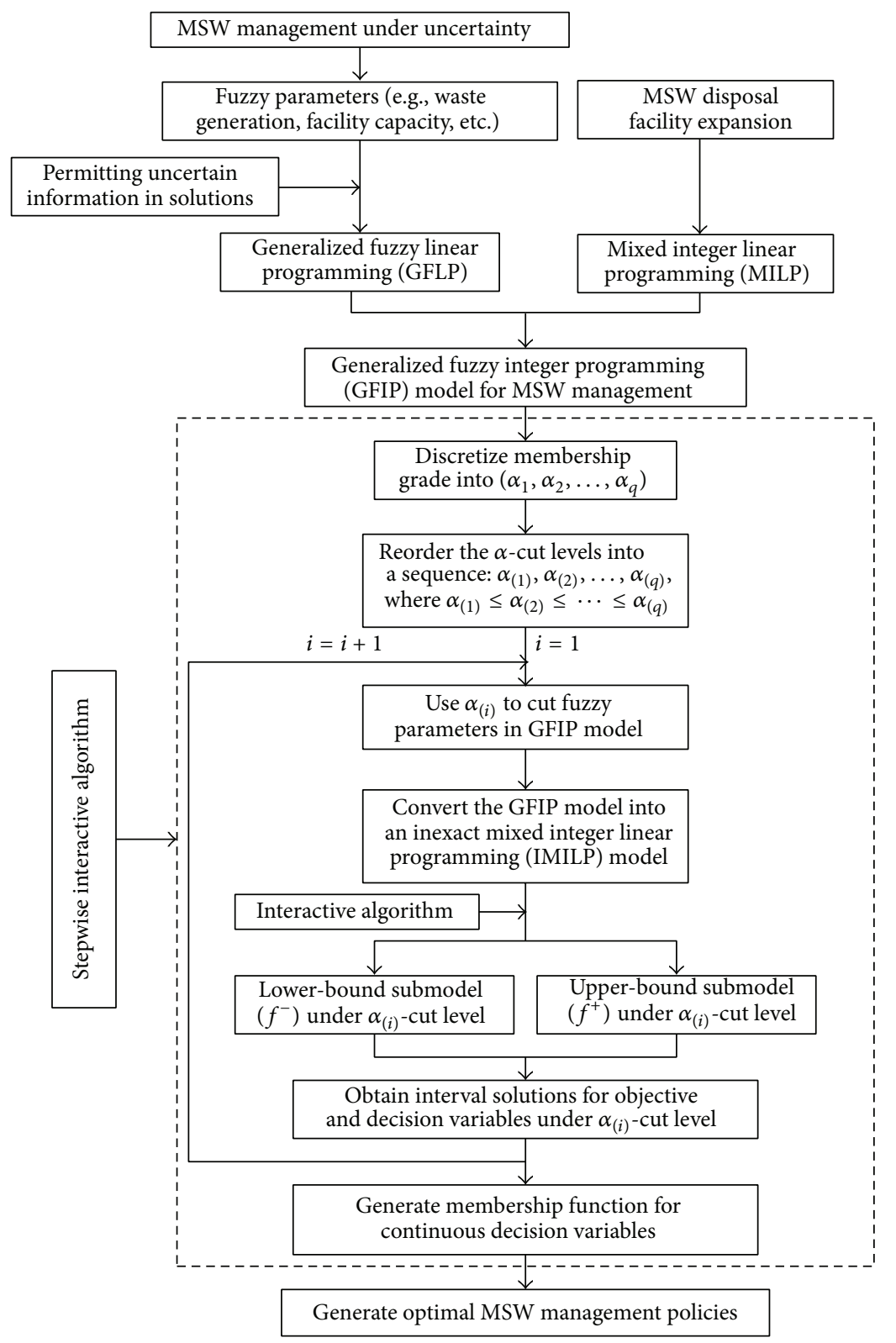

FIgURE 1: The schematic of the GFIP model for MSW management.

allocated to the landfill, with a waste flow of $(30,25$, and 25) tonne/day. The $(30,25$, and 25$)$ indicates a triangular fuzzy number, with 30,5 , and 55 as its most possible value and lower and upper bound, respectively. The fuzzy characteristic of the waste flow to the landfill indicates that the variation in the waste generation rate of city 1 would be handled through landfilling. The waste disposal scheme for city 2 is much different from that of city 1 . The waste-treatment demand of city 2 can be satisfied through landfilling. Consequently, no waste would flow to WTE in this period. Conversely, all of the generated waste in city 3 would be shipped to WTE in spite of its variation in waste generation rate.

The waste allocation schemes for three cities in period 2 would be similar to those in period 1 . The majority of waste from city 1 would be allocated to WTE, with the residues being shipped to landfill. However, compared with the waste flows in period 1 , more waste would be transported to WTE in period 2 as a result of temporally increasing waste generation rate. Moreover, the amount of waste allocated to WTE from city 1 would fluctuate within small intervals under low plausibilities $((225,236.6)$ and $(225,229.9)$ tonne/day under $\alpha=0$ and 0.3 , resp.). This is because the waste generation rate would vary within significant ranges under low plausibilities. Meanwhile, landfill would be the only choice to satisfy the waste-treatment demand of city 2, while all waste from city 3 would be shipped to WTE.

The waste-flow patterns would be changed significantly in period 3. All waste from the three cities would be delivered to the landfill due to its lower operation cost. In detail, waste flows shipped to the landfill from cities 1, 2, and 3 would be 
The upper bound of waste flow under different $\alpha$-cut values

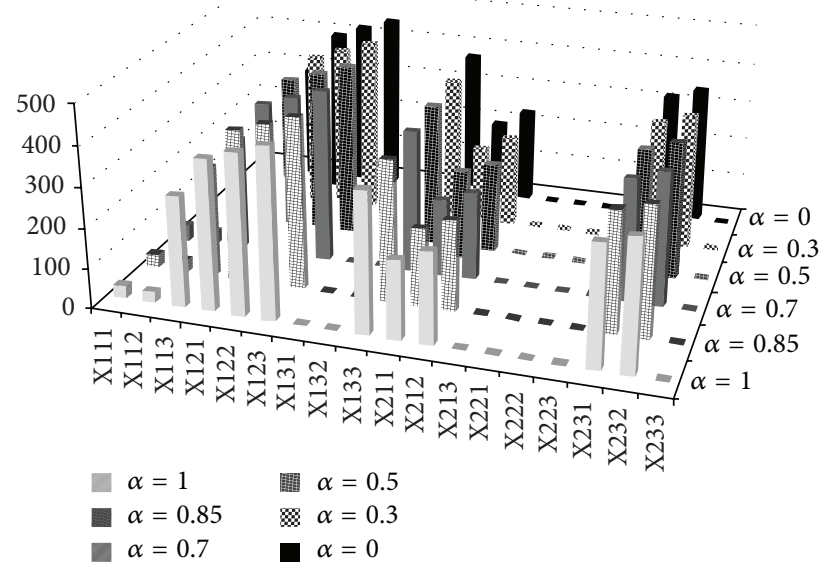

FIGURE 2: The upper bounds of waste flows under different $\alpha$-cut levels.

(250, 275, and 300), (400, 425, and 450), and (325, 350, and $375)$ tonne/day, respectively. Also, the (250, 275, and 300), $(400,425$, and 450$)$ and $(325,350$, and 375) indicates three triangular fuzzy numbers, reflecting uncertainty in resulting solutions derived from uncertain inputs.

Since parameters in model (10a)-(10i) are expressed as fuzzy sets, the fluctuating ranges of these inputs would be varied under different plausibilities ( $\alpha$-cut levels) and thus result in variations in the generated solutions. For example, under $\alpha=0$ (the lowest plausibility degree), the amount of waste allocated to the landfill from city 1 [denoted as $\left(X_{111}\right)_{\alpha}^{ \pm}$] would be $[5,55]$ tonne/day; in comparison, under $\alpha=1$ (the highest plausibility degree), this waste flow would be 30 tonne/day. As the value of $\alpha$-cut level increases from 0 to 1 , the lower bound of $\left(X_{111}\right)_{\alpha}^{ \pm}$would also increase (i.e., 12.5, 17.5, 22.5 , and 26.25 tonne/day under $\alpha=0.3,0.5,0.7$, and 0.85 , resp.), while the upper bound of $\left(X_{111}\right)_{\alpha}^{ \pm}$would decrease (i.e., $47.5,42.5,37.5$, and 33.75 tonne/day under $\alpha=0.3,0.5,0.7$, and 0.85 , resp.). Figures 2 and 3 show the lower and upper bounds of waste-flow patterns under different $\alpha$-cut levels. They indicate that solutions of waste diversion schemes from three cities would vary as the variation in $\alpha$-cut levels. The lower bound would increase and the upper bound would decrease when the $\alpha$-cut level increases from 0 to 1 . Such variations in waste-flow patterns would stem from the input fuzziness of model (10a)-(10i).

Multiple capacity expanding options are considered in response to fuzzy characteristics of the input parameters. Table 4 shows capacity expanding options for the landfill. Different capacity options would be applied under different plausibilities ( $\alpha$-cut levels). In detail, the landfill would be expanded in period 1 under $\alpha=0$. When $\alpha=0$, model (10a)-(10i) would consider all possible values of the waste generation rates; as a result, the landfill would be expanded in period 1 to tackle the variations in waste generation rates. As the $\alpha$-cut level increases, uncertainties of the inputs would decrease, leading to adaptation of expanding option for the landfill. When $\alpha=0.3$, landfill expansion would be applied in period 1 under demanding conditions (i.e., the
The lower bounds of waste flow under different $\alpha$-cut values

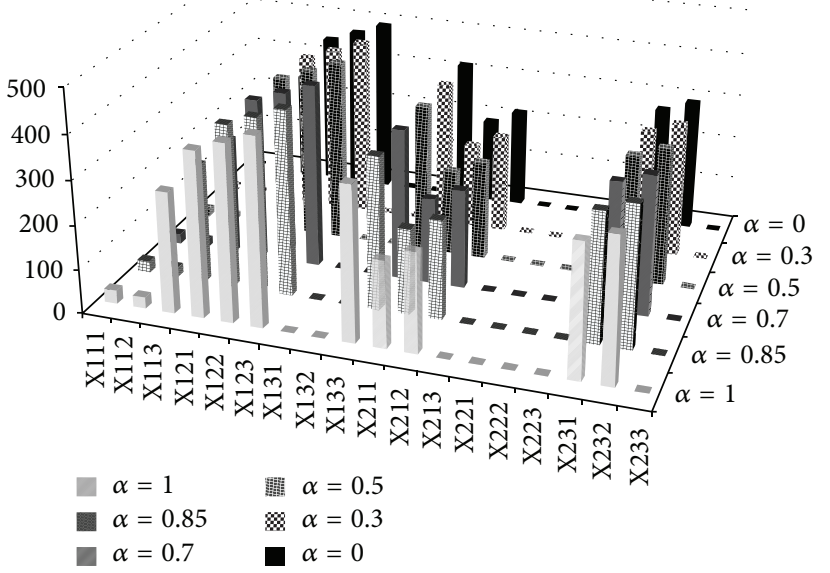

FIgURE 3: The lower bounds of waste flows under different $\alpha$-cut levels.

capacity of landfill achieves its lower bound and the waste generation rates reach their upper bounds). This means that the existing capacity of landfill would be sufficient to dispose of the solid waste under advantageous conditions (i.e., the capacity of landfill achieves its upper bound and the waste generation rates reach their lower bounds). However, when $\alpha$-cut level increases to 0.5 , the landfill would require an expansion in period 2 under advantageous conditions due to the increase in the lower bounds of waste generation rates and the decrease in the upper bound of landfill capacity. Under $\alpha=0.7$, expansion option of the landfill would be similar to that under $\alpha=0.3$, except the option being applied in period 2. As shown in Table 4, when $\alpha \geq 0.85$, the landfill would be expanded in period 2 under both demanding and advantageous conditions. In brief, the results in Table 4 suggest that (i) expansion of the landfill in period 1 leads to low risk but high system cost and (ii) the landfill at least is expanded in period 2 .

As shown in Table 5, the WTE facility would generally be expanded in periods 1 and 2 . In period 1, option 1 (i.e., 150 tonne/day) would be applied for WTE, regardless of the impacts of uncertain inputs. However, in period 2, the capacity expanding options would be influenced significantly by system uncertainty. The WTE facility would be expanded with different options under different plausibilities in this period. When $\alpha=0$, option 1 is considered to be suitable for WTE under advantageous conditions, while option 3 is chosen under demanding conditions. As the $\alpha$-cut level varies between 0.3 and 0.7 , options 1 and 2 are applicable for WTE under advantageous and demanding conditions. Furthermore, as shown in Table 5, the WTE facility would at least be expanded with option 2 in period 2.

Based on the waste-flow diversion schemes under selected $\alpha$-cut levels (as shown in Table 3 ), we can approximate their membership functions through statistical regression. Figure 4 shows the membership functions for the wasteflow schemes. It indicates that these membership functions can be well fitted based on the results in Table 3. However, 
for the variables indicating facility expansion options, it can hardly obtain their membership function since they are binary variables. But decision makers can still generate appropriate waste-flow diversion schemes and facility expansion options based on Figure 4 and Tables 4 and 5. For example, if a decision maker wants to identify the waste management policy under an $\alpha$-cut level of 0.6 , the waste-flow patterns can be obtained through the membership functions in Figure 4 (e.g., $\left(X_{111}\right)_{0.6}^{-}=(0.6+0.2) / 0.04=20,\left(X_{111}\right)_{0.6}^{+}=(2.2-$ $0.6) / 0.4=40)$; the facility expansion options can be adopted based on results under $\alpha=0.5$ and 0.7 in Tables 4 and 5 .

Table 2 also provides the total system costs (denoted as the objective function) obtained from the GFIP model under six $\alpha$-cut levels. The results suggest that different plausibilities of uncertain inputs lead to varied system costs. The lower bounds of the objective function correspond to advantageous conditions, while the upper bounds are associated with demanding conditions. When $\alpha=0$, the system cost would be $\$[6.09,8.02] \times 10^{9}$. Under $\alpha=0.3$, the lower bound of system cost would be $\$ 6.18 \times 10^{9}$ and the upper bound would be $\$ 7.27 \times 10^{9}$, leading to a fluctuating interval of $\$[6.18,7.27] \times 10^{9}$ for system cost. However, the value of the objective function does not necessarily hold such a characteristic that as the $\alpha$-cut level increases, the lower bound increases and the upper bound decreases (as shown in Figure 5). For example, the lower bound of the objective function under $\alpha=0.5$ and 0.7 is $\$ 6.24 \times 10^{9}$ and $\$ 3.8 \times$ $10^{9}$, respectively. This is due to the variation in capacityexpanding options under these two $\alpha$-cut levels. When $\alpha=0.5$, capacity-expanding for landfill is considered under both demanding and advantageous conditions; in contrast, the landfill would not be expanded under advantageous conditions when $\alpha=0.7$, which leads to a significant decrease of the objective function value.

Generally speaking, the conventional inexact mixedinteger linear programming (IMILP) method would consider uncertain parameters with known lower and upper bounds. For the IMILP method, it cannot consider any distributional information between the lower and upper bounds. In this study, the solutions of GFIP model under $\alpha=0$ are identical to the solutions obtained from the IMILP model. As shown in Tables 3 to 5, the solutions of IMILP model can only provide interval values for waste allocation schemes and neglect distributional information within these intervals. Particularly, when distributional information is available for uncertain inputs, the IMILP method can hardly reflect the relationships between uncertainty of the inputs and the resulting solutions. Conversely, the GFIP method can not only provide fluctuating intervals for waste-flow allocation schemes (i.e., lower and upper bounds) as well as corresponding capacity expanding options for waste-treatment facilities but also afford plausibilities for such waste management alternatives.

\section{Conclusions}

In this study, a generalized fuzzy integer programming (GFIP) method was developed for solid waste management under uncertainty. The developed GFIP could deal with uncertainties expressed as fuzzy sets that exist in the constraints' left- and right-sides and the objective function. A stepwise interactive algorithm (SIA) was proposed to solve the GFIP model and generate solutions expressed as fuzzy sets. The SIA firstly discretized the membership function grade into a set of $\alpha$-cut levels. Then the GFIP model was converted into a series of IMILP submodels. The interactive algorithm proposed by Huang et al. [3] was applied to solve the IMILP submodels and generate interval solutions under each $\alpha$-cut level. The membership functions for fuzzy continuous variables were finally obtained through statistical regression method based on those interval solutions.

The developed method was applied to a case of municipal solid waste management to illustrate the applicability of GFIP method. The solutions for binary variables, which were obtained as discrete fuzzy sets, provided different capacity expansion alternatives for waste-treatment facilities under different plausibilities of uncertain inputs. The solutions for continuous variables, which were expressed as fuzzy sets with known membership functions, provided optimal schemes for waste-flow allocations. These solutions were used for generating decision alternatives and thus helping decision makers to identify desired policies for MSW management under uncertainty. Although the case study in this study is just a hypothetical one, this case involved the main factors (e.g., transportation, operation, and capacity expansion costs) in MSW management. Consequently, the developed GFIP method can also be applied to real-world MSW management problems. Through the hypothetical (MSW) case study, the following advantages of the GFIP approach were presented: (i) GFIP could deal with fuzzy parameters with any kind of membership function shape; (ii) the solution process of GFIP would not generate complicated intermediate submodels; (iii) the computational requirement was reasonable; and (iv) the solutions of GFIP exhibited some distributional information, which was helpful for decision making. The above advantages indicated the usefulness and applicability of the developed GFIP approach in real-world MSW management problem.

Compared with the inexact mixed-integer linear programming (IMILP) method, uncertainties presented as fuzzy sets were incorporated within the GFIP's optimization processes. Solutions obtained from GFIP method specified some distributional information, which contained not only the lower and upper bounds with associated plausibility degrees but also the most possible values.

The developed GFIP could deal with various fuzzy sets with known membership functions, regardless of the shapes of these functions. However, it had difficulties in dealing with other kinds of uncertainties expressed as probabilistic distributions. Therefore, improvement for GFIP was further desired to enhance its capability of dealing with multiple uncertainties through incorporating interval programming or stochastic programming into the GFIP framework. Besides, the developed GFIP method cannot be used for evaluating the detailed effects of interactions among multiple uncertain inputs. Consequently, the factorial analysis method could then be integrated into the GFIP framework to address the above issue. 

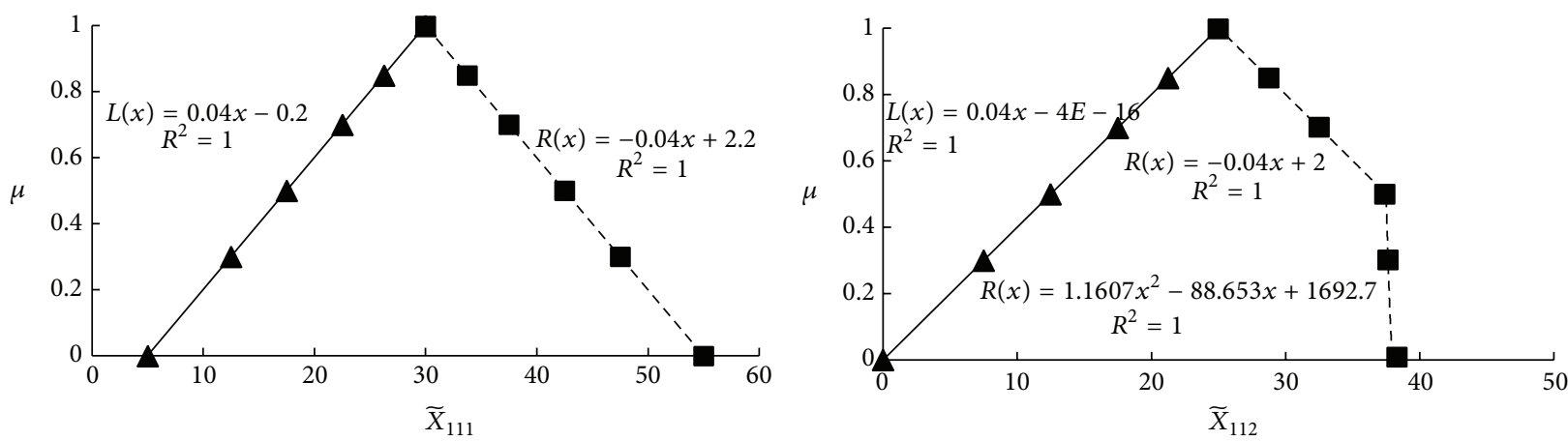

\ Lower bound

- Upper bound

Lower bound

- - - Upper bound

- Lower bound

Lower bound

- Upper bound

- - - Upper bound
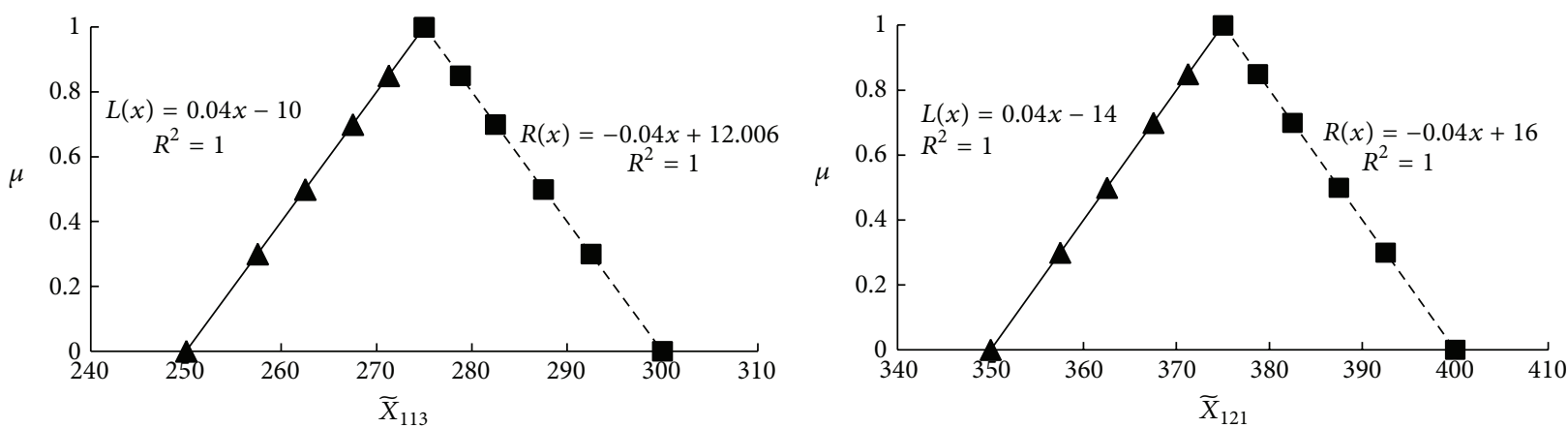

\ Lower bound

- Upper bound

_ Lower bound --- Upper bound

\ Lower bound - Upper bound

_ Lower bound --- Upper bound
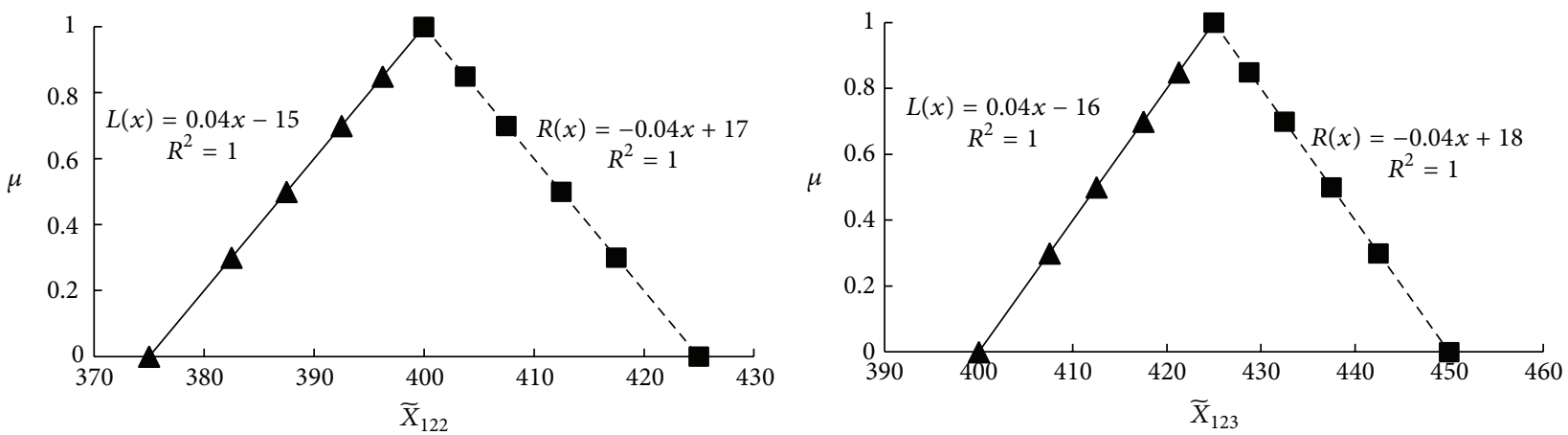

\ Lower bound - Upper bound

_ Lower bound --- Upper bound

\ Lower bound - Upper bound

_ Lower bound -- - Upper bound
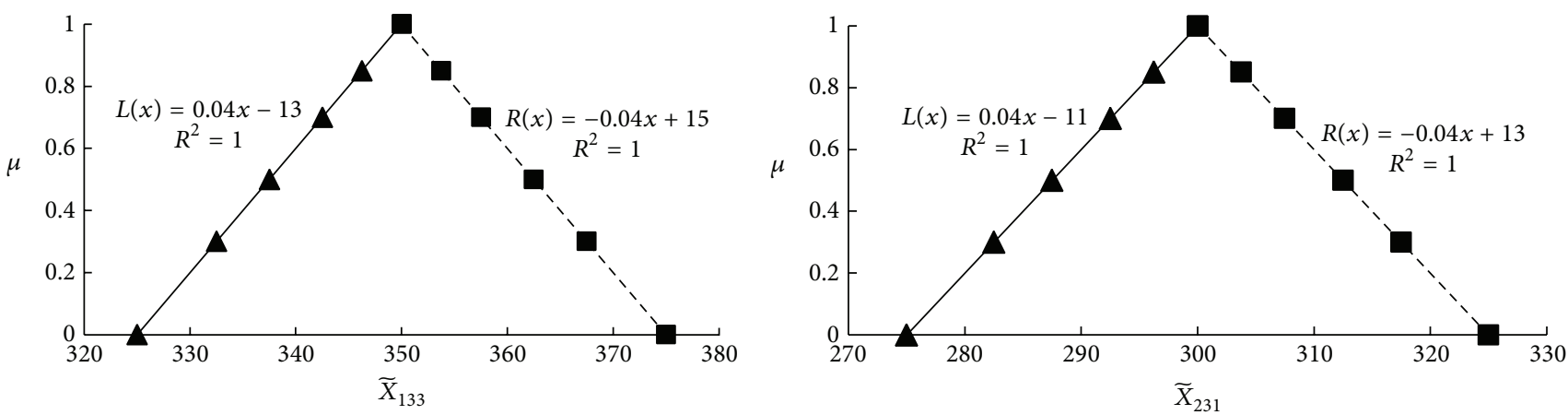

\ Lower bound - Upper bound

\ Lower bound

- Upper bound

Lower bound

- - Upper bound

(a)

FIGURE 4: Continued. 


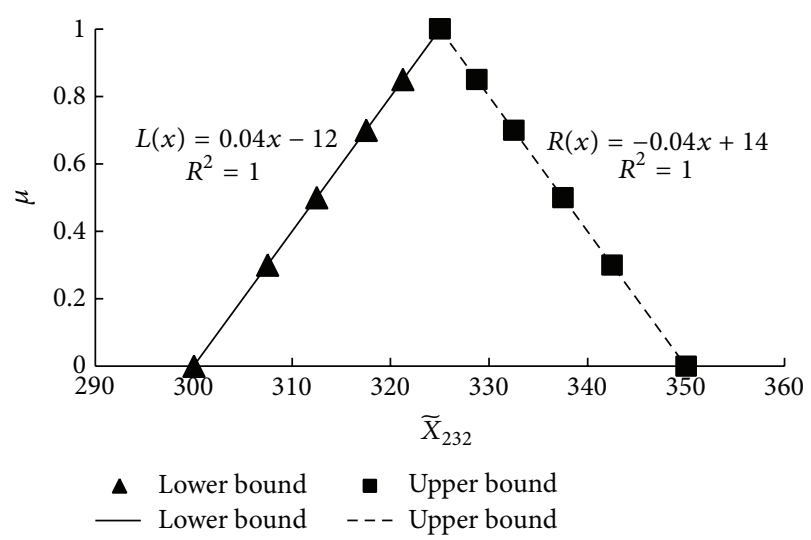

(b)

FIgURe 4: The membership functions for fuzzy variables.

TABLE 3: Solutions for the GFIP under each $\alpha$-cut level.

\begin{tabular}{|c|c|c|c|c|c|c|}
\hline \multirow{2}{*}{ Waste allocation } & \multicolumn{6}{|c|}{$\alpha$-cut level } \\
\hline & 0 & 0.3 & 0.5 & 0.7 & 0.85 & 1 \\
\hline$\overline{\left(X_{111}\right)_{\alpha}^{ \pm}}$ & {$[5,55]$} & {$[12.5,47.5]$} & {$[17.5,42.5]$} & {$[22.5,37.5]$} & {$[26.25,33.75]$} & 30 \\
\hline$\left(X_{121}\right)_{\alpha}^{ \pm}$ & {$[350,400]$} & {$[357.5,392.5]$} & {$[362.5,387.5]$} & {$[367.5,382.5]$} & {$[371.25,378.75]$} & 375 \\
\hline$\left(X_{131}\right)_{\alpha}^{ \pm}$ & 0 & 0 & 0 & 0 & 0 & 0 \\
\hline$\left(X_{112}\right)_{\alpha}^{ \pm}$ & {$[0,38.4]$} & {$[7.5,37.6]$} & {$[12.5,37.5]$} & {$[17.5,32.5]$} & {$[21.25,28.75]$} & 25 \\
\hline$\left(X_{122}\right)_{\alpha}^{ \pm}$ & {$[375,425]$} & {$[382.5,417.5]$} & {$[387.5,412.5]$} & {$[392.5,407.5]$} & {$[396.25,403.75]$} & 400 \\
\hline$\left(X_{132}\right)_{\alpha}^{ \pm}$ & 0 & 0 & 0 & 0 & 0 & 0 \\
\hline$\left(X_{113}\right)_{\alpha}^{ \pm}$ & {$[250,300]$} & {$[257.5,292.5]$} & {$[262.5,287.5]$} & {$[267.5,282.5]$} & {$[271.25,278.75]$} & 275 \\
\hline$\left(X_{123}\right)_{\alpha}^{ \pm}$ & {$[400,450]$} & {$[407.5,442.5]$} & {$[412.5,437.5]$} & {$[417.5,432.5]$} & {$[421.25,428.75]$} & 425 \\
\hline$\left(X_{133}\right)_{\alpha}^{ \pm}$ & {$[325,375]$} & {$[332.5,367.5]$} & {$[337.5,362.5]$} & {$[342.5,357.5]$} & {$[346.25,353.75]$} & 350 \\
\hline$\left(X_{211}\right)_{\alpha}^{ \pm}$ & 195 & 195 & 195 & 195 & 195 & 195 \\
\hline$\left(X_{221}\right)_{\alpha}^{ \pm}$ & 0 & 0 & 0 & 0 & 0 & 0 \\
\hline$\left(X_{231}\right)_{\alpha}^{ \pm}$ & {$[275,325]$} & {$[282.5,317.5]$} & {$[287.5,312.5]$} & {$[292.5,307.5]$} & {$[296.25,303.75]$} & 300 \\
\hline$\left(X_{212}\right)_{\alpha}^{ \pm}$ & {$[225,236.6]$} & {$[225,229.9]$} & 225 & 225 & 225 & 225 \\
\hline$\left(X_{222}\right)_{\alpha}^{ \pm}$ & 0 & 0 & 0 & 0 & 0 & 0 \\
\hline$\left(X_{232}\right)_{\alpha}^{ \pm}$ & {$[300,350]$} & {$[307.5,342.5]$} & {$[312.5,337.5]$} & {$[317.5,332.5]$} & {$[321.25,328.75]$} & 325 \\
\hline$\left(X_{213}\right)_{\alpha}^{ \pm}$ & 0 & 0 & 0 & 0 & 0 & 0 \\
\hline$\left(X_{223}\right)_{\alpha}^{ \pm}$ & 0 & 0 & 0 & 0 & 0 & 0 \\
\hline$\left(X_{233}\right)_{\alpha}^{ \pm}$ & 0 & 0 & 0 & 0 & 0 & 0 \\
\hline$(f)_{\alpha}^{ \pm}\left(\times 10^{9}\right)$ & {$[6.09,8.02]$} & {$[6.18,7.27]$} & {$[6.24,7.2]$} & {$[3.8,7.14]$} & {$[7,7.1]$} & 7.05 \\
\hline
\end{tabular}

\section{Appendix}

Definition $A .1$ (fuzzy set). Let $X$ denote a universal set. Then a fuzzy set $\widetilde{A}$ in $X$ can be defined by a membership function as follows:

$$
\mu_{\widetilde{A}}: X \rightarrow[0,1]
$$

For each $x \in X$, the value of $\mu_{\widetilde{A}}(x)$ shows the grade (or degree) of membership of the element $x$ of $X$ in fuzzy set $\widetilde{A}$.

Definition A.2 ( $\alpha$-cut). Given a fuzzy set $\widetilde{A}$ defined on $X$ and a particular number $\alpha$ in the unit interval $[0,1]$, the $\alpha$-cut of $\widetilde{A}$, denoted as $[\widetilde{A}]_{\alpha}$, is a crisp set that consists of all elements of $X$ whose membership degrees in $\widetilde{A}$ are greater than or equal to $\alpha$ :

$$
[\widetilde{A}]_{\alpha}=\left\{x \mid \mu_{\widetilde{A}}(x) \geq \alpha, \quad \alpha \in[0,1]\right\} .
$$

Definition A.3 (fuzzy number). A fuzzy number is a convex normalized fuzzy set in the real number whose membership function is piecewise continuous. 
TABLE 4: Capacity expanding options for the landfill under each $\alpha$-cut level.

\begin{tabular}{lcccccc}
\hline Expanding options & \multicolumn{3}{c}{$\alpha$-cut level } & 0.5 & 0.85 \\
\hline$\left(Y_{1}\right)_{\alpha}^{ \pm}$ & 0 & 0.3 & {$[0,1]$} & {$[0,1]$} & {$[0,0]$} & 0 \\
$\left(Y_{2}\right)_{\alpha}^{ \pm}$ & {$[1,1]$} & 0 & {$[1,0]$} & {$[0,1]$} & 0 \\
$\left(Y_{3}\right)_{\alpha}^{ \pm}$ & 0 & 0 & 0 & 0 & $1,1]$ & 0 \\
\hline
\end{tabular}

TABLE 5: Capacity expanding options for the WTE facility under each $\alpha$-cut level.

\begin{tabular}{lccccc}
\hline Waste allocation & & \multicolumn{2}{c}{$\alpha$-cut level } & 0.7 & 1 \\
\hline$\left(Z_{11}\right)_{\alpha}^{ \pm}$ & 0 & 0.3 & 0.5 & 1 & 1 \\
$\left(Z_{21}\right)_{\alpha}^{ \pm}$ & 1 & 1 & 0 & 0 & 0.85 \\
$\left(Z_{31}\right)_{\alpha}^{ \pm}$ & 0 & 0 & 0 & 0 & 0 \\
$\left(Z_{12}\right)_{\alpha}^{ \pm}$ & 0 & 0 & {$[1,0]$} & 0 & 0 \\
$\left(Z_{22}\right)_{\alpha}^{ \pm}$ & {$[1,0]$} & {$[1,0]$} & {$[0,1]$} & 0 & 0 \\
$\left(Z_{32}\right)_{\alpha}^{ \pm}$ & 0 & {$[0,1]$} & 0 & 0 & 0 \\
$\left(Z_{13}\right)_{\alpha}^{ \pm}$ & {$[0,1]$} & 0 & 0 & 0 & 0 \\
$\left(Z_{23}\right)_{\alpha}^{ \pm}$ & 0 & 0 & 0 & 0 & 0 \\
$\left(Z_{33}\right)_{\alpha}^{ \pm}$ & 0 & 0 & 0 & 0 & 0 \\
\hline
\end{tabular}

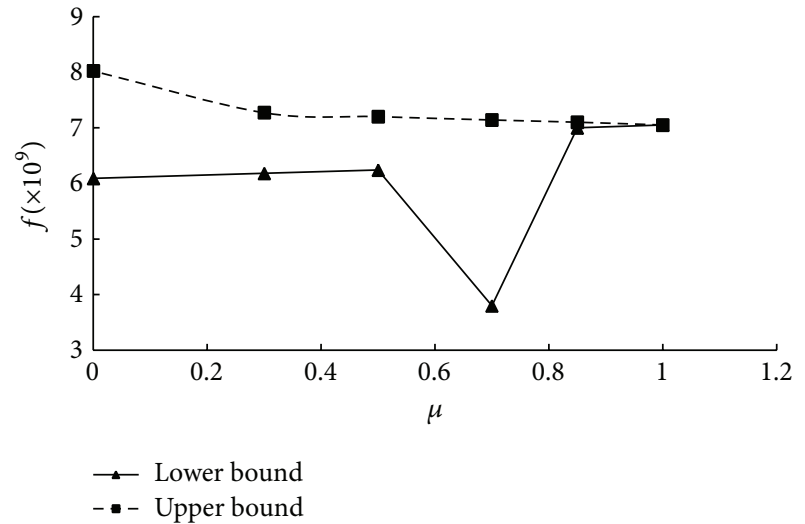

FIGURE 5: The objective function values under different $\alpha$-cut levels.

Definition $A .4$ ( $L-R$ fuzzy numbers). A fuzzy number $M$ is a so-called $L-R$ fuzzy, $M=(m, \alpha, \beta)_{L R}$, if the corresponding membership function satisfies for all $x \in R$ :

$$
\mu_{M}(x)= \begin{cases}L\left(\frac{m-x}{\alpha}\right) & \text { for } m-\alpha \leq x \leq m \\ R\left(\frac{x-m}{\beta}\right) & \text { for } m \leq x \leq m+\beta \\ 0 & \text { else }\end{cases}
$$

where $m$ is the mean value of $M ; \alpha>0$ and $\beta>0$ are left and right spread, respectively; $L$ and $R$ are called the left and right shape function, respectively, which are strictly decreasing continuous functions from $[0,1]$ to $[0,1]$ such that $L(0)=R(0)=1$ and $L(1)=R(1)=0$. If $L(x)$ and $R(x)$ are linear functions, then the corresponding $L-R$ fuzzy number is considered as a triangular fuzzy number.
Definition A.5. Let $F_{0}(R)$ denote the set of all fuzzy numbers in $R$. For any $\tilde{a} \in F_{0}(R)$, an $\alpha$-cut of $\tilde{a}$ can be expressed as a closed interval:

$$
a_{\alpha}^{ \pm}=\left[a_{\alpha}^{-}, a_{\alpha}^{+}\right], \quad \text { for any } \alpha \in[0,1], a_{\alpha}^{-} \leq a_{\alpha}^{+} .
$$

Remark A.6. For any $\tilde{a} \in F_{0}(R)$, suppose two $\alpha$-cut levels $\alpha_{1}$, $\alpha_{2}$ are selected to cut $\tilde{a}$; then these two $\alpha$-cuts can be formulated as:

$$
a_{\alpha_{1}}^{ \pm}=\left[a_{\alpha_{1}}^{-}, a_{\alpha_{1}}^{+}\right], \quad a_{\alpha_{2}}^{ \pm}=\left[a_{\alpha_{2}}^{-}, a_{\alpha_{2}}^{+}\right] .
$$

If $\alpha_{1} \geq \alpha_{2}$, we have:

$$
a_{\alpha_{1}}^{ \pm} \subseteq a_{\alpha_{2}}^{ \pm}, \quad \text { namely } a_{\alpha_{1}}^{-} \geq a_{\alpha_{2}}^{-}, a_{\alpha_{1}}^{+} \leq a_{\alpha_{2}}^{+} .
$$

Definition A.7. For $a_{\alpha}^{ \pm}=\left[a_{\alpha}^{-}, a_{\alpha}^{+}\right]$and $b_{\alpha}^{ \pm}=\left[b_{\alpha}^{-}, b_{\alpha}^{+}\right]$, we can define:

(1) $a_{\alpha}^{ \pm}+b_{\alpha}^{ \pm}=\left[a_{\alpha}^{-}, a_{\alpha}^{+}\right]+\left[b_{\alpha}^{-}, b_{\alpha}^{+}\right]=\left[a_{\alpha}^{-}+b_{\alpha}^{-}, a_{\alpha}^{+}+b_{\alpha}^{+}\right]$.

(2) $a_{\alpha}^{ \pm}-b_{\alpha}^{ \pm}=\left[a_{\alpha}^{-}, a_{\alpha}^{+}\right]-\left[b_{\alpha}^{-}, b_{\alpha}^{+}\right]=\left[a_{\alpha}^{-}-b_{\alpha}^{+}, a_{\alpha}^{+}-b_{\alpha}^{-}\right]$.

(3) $a_{\alpha}^{ \pm} \cdot b_{\alpha}^{ \pm}=\left[a_{\alpha}^{-}, a_{\alpha}^{+}\right] \cdot\left[b_{\alpha}^{-}, b_{\alpha}^{+}\right]=\left[a_{\alpha}^{-} b_{\alpha}^{-} \wedge a_{\alpha}^{-} b_{\alpha}^{+} \wedge a_{\alpha}^{+} b_{\alpha}^{-} \wedge\right.$ $\left.a_{\alpha}^{+} b_{\alpha}^{+}, a_{\alpha}^{-} b_{\alpha}^{-} \vee a_{\alpha}^{-} b_{\alpha}^{+} \vee a_{\alpha}^{+} b_{\alpha}^{-} \vee a_{\alpha}^{+} b_{\alpha}^{+}\right]$.

(4) The order relation " $\leq$ " is defined by:

$$
\left[a_{\alpha}^{-}, a_{\alpha}^{+}\right] \leq\left[b_{\alpha}^{-}, b_{\alpha}^{+}\right] \quad \text { iff } a_{\alpha}^{-} \leq b_{\alpha}^{-}, a_{\alpha}^{+} \leq b_{\alpha}^{+} .
$$

(5) Let $\left[a_{\alpha_{i}}^{-}, a_{\alpha_{i}}^{+}\right] \subset R, i \in I$; I is the index set; then:

$$
\begin{aligned}
& \hat{i \in I}_{i}\left[a_{\alpha_{i}}^{-}, a_{\alpha_{i}}^{+}\right]=\left[\wedge a_{i \in I}^{-}, a_{\alpha_{i}}, \wedge_{i \in I}^{+} a_{\alpha_{i}}^{+}\right], \quad \text { if } \wedge_{i \in I} a_{\alpha_{i}}^{-}>-\infty \text {, } \\
& \underset{i \in I}{\vee}\left[a_{\alpha_{i}}^{-}, a_{\alpha_{i}}^{+}\right]=\left[\underset{i \in I}{\vee} a_{\alpha_{i}}^{-}, \vee_{i \in I}^{\vee} a_{\alpha_{i}}^{+}\right], \quad \text { if } \underset{i \in I}{\vee} a_{\alpha_{i}}^{+}<\infty \text {. }
\end{aligned}
$$

Definition A.8. Let $\left\{\tilde{a}_{i} \mid i \in I\right\} \subset F_{0}(R), \alpha \in[0,1]$; then 
(1) $\tilde{f}=\wedge_{i \in I} \widetilde{a}_{i}$ is defined by a fuzzy number $\tilde{a}_{i} \in F_{0}(R)$ such that $f_{\alpha}=\wedge_{i \in I}\left(a_{i}\right)_{\alpha}$;

(2) $\tilde{g}=\vee_{i \in I} \tilde{a}_{i}$ is defined by a fuzzy number $\tilde{a}_{i} \in F_{0}(R)$ such that $g_{\alpha}=\vee_{i \in I}\left(a_{i}\right)_{\alpha}$.

Definition A.9. Let $\tilde{a}, \tilde{b} \in F_{0}(R)$. Then for any $\alpha \in(0,1]$, we have:

$$
(a * b)_{\alpha}=a_{\alpha} * b_{\alpha}
$$

where $*$ may be any continuous algebraic operation.

\section{Conflict of Interests}

The authors declare that there is no conflict of interests regarding the publication of this paper.

\section{Acknowledgments}

This research was supported by the Major Project Program of the Natural Sciences Foundation (51190095), the Program for Innovative Research Team in University (IRT1127), and the MOE Key Project Program (311013). The authors are extremely grateful to the editor and the anonymous reviewers for their insightful comments and suggestions.

\section{References}

[1] Y. P. Li and G. H. Huang, "Dynamic analysis for solid waste management systems: An inexact multistage integer programming approach," Journal of the Air \& Waste Management Association, vol. 59, no. 3, pp. 279-292, 2009.

[2] B. W. Baetz, "Capacity planning for waste management systems," Civil Engineering Systems, vol. 7, pp. 229-235, 1990.

[3] G. H. Huang, B. W. Baetz, and G. G. Patry, "Grey integer programming: an application to waste management planning under uncertainty," European Journal of Operational Research, vol. 83, no. 3, pp. 594-620, 1995.

[4] E. Davila, N. Chang, and S. Diwakaruni, "Landfill space consumption dynamics in the Lower Rio Grande Valley by grey integer programming-based games," Journal of Environmental Management, vol. 75, no. 4, pp. 353-365, 2005.

[5] Y. R. Fan, G. H. Huang, P. Guo, and A. L. Yang, "Inexact two-stage stochastic partial programming: application to water resources management under uncertainty," Stochastic Environmental Research and Risk Assessment, vol. 26, no. 2, pp. 281-293, 2012.

[6] Q. Hu, G. H. Huang, Y. P. Cai, and W. Sun, "Planning of electric power generation systems under multiple uncertainties and constraint-violation levels," Journal of Environmental Informatics, vol. 23, no. 1, pp. 55-64, 2014.

[7] Y. P. Li and G. H. Huang, "An inexact two-stage mixed integer linear programming method for solid waste management in the City of Regina," Journal of Environmental Management, vol. 81, no. 3, pp. 188-209, 2006.

[8] M. Delgado, J. L. Verdegay, and M. A. Vila, "A general model for fuzzy linear programming," Fuzzy Sets and Systems, vol. 29, no. 1, pp. 21-29, 1989.

[9] M. Delgado, F. Herrera, J. L. Verdegay, and M. A. Vila, "Postoptimality analysis on the membership functions of a fuzzy linear programming problem," Fuzzy Sets and Systems, vol. 53, no. 3, pp. 289-297, 1993.

[10] H. Rommelfanger, "Fuzzy linear programming and applications," European Journal of Operational Research, vol. 92, no. 3, pp. 512-527, 1996.

[11] H. R. Maleki, M. Tata, and M. Mashinchi, "Linear programming with fuzzy variables," Fuzzy Sets and Systems, vol. 109, no. 1, pp. 21-33, 2000.

[12] Y. R. Fan, G. H. Huang, Y. P. Li, M. F. Cao, and G. H. Cheng, "A fuzzy linear programming approach for municipal solid-waste management under uncertainty," Engineering Optimization, vol. 41, no. 12, pp. 1081-1101, 2009.

[13] A. K. Srivastava and A. K. Nema, "Fuzzy parametric programming model for integrated solid waste management under uncertainty," Journal of Environmental Engineering, vol. 137, no. 1, pp. 69-83, 2011.

[14] A. K. Srivastava and A. K. Nema, "Fuzzy parametric programming model for multi-objective integrated solid waste management under uncertainty," Expert Systems with Applications, vol. 39, no. 5, pp. 4657-4678, 2012.

[15] T. Y. Xu and X. S. Qin, "Solving water quality management problem through combined genetic algorithm and fuzzy simulation," Journal of Environmental Informatics, vol. 22, no. 1, pp. 39-48, 2013.

[16] J. J. Buckley and T. Feuring, "Evolutionary algorithm solution to fuzzy problems: fuzzy linear programming," Fuzzy Sets and Systems, vol. 109, no. 1, pp. 35-53, 2000.

[17] S. M. Hashemi, M. Modarres, E. Nasrabadi, and M. M. Nasrabadi, "Fully fuzzified linear programming, solution and duality," Journal of Intelligent and Fuzzy Systems, vol. 17, no. 3, pp. 253-261, 2006.

[18] F. Hosseinzadeh Lotfi, T. Allahviranloo, M. Alimardani Jondabeh, and L. Alizadeh, "Solving a full fuzzy linear programming using lexicography method and fuzzy approximate solution," Applied Mathematical Modelling, vol. 33, no. 7, pp. 3151-3156, 2009.

[19] Y. Fan, G. Huang, and A. Veawab, "A generalized fuzzy linear programming approach for environmental management problem under uncertainty," Journal of the Air \& Waste Management Association, vol. 62, no. 1, pp. 72-86, 2012.

[20] Y. R. Fan, G. H. Huang, and A. L. Yang, "Generalized fuzzy linear programming for decision making under uncertainty: feasibility of fuzzy solutions and solving approach," Information Sciences, vol. 241, pp. 12-27, 2013.

[21] A. Kumar, J. Kaur, and P. Singh, "A new method for solving fully fuzzy linear programming problems," Applied Mathematical Modelling, vol. 35, no. 2, pp. 817-823, 2011.

[22] H. Zimmermann, Fuzzy Set Theory-and Its Applications, Kluwer-Nijhoff, Dordrecht, The Netherlands, 1985.

[23] J. J. Buckley and L. J. Jowers, Monte Carlo Methods in Fuzzy Optimization, Studies in Fuzziness and Soft Computing, Springer, Berlin, Germany, 2008.

[24] Y. J. Lai and C. L. Hwang, Fuzzy Mathematical Programming, Springer, Berlin, Germany, 1992.

[25] A. Kaufmann and M. Cupta, Fuzzy Mathematical Models in Engineering and Many Science, North Holland Publishing, Amsterdam, The Netherlands, 1988.

[26] E. E. Ammar, "On solutions of fuzzy random multiobjective quadratic programming with applications in portfolio problem," Information Sciences, vol. 178, no. 2, pp. 468-484, 2008. 
[27] M. Dehghan, B. Hashemi, and M. Ghatee, "Computational methods for solving fully fuzzy linear systems," Applied Mathematics and Computation, vol. 179, no. 1, pp. 328-343, 2006.

[28] M. Dehghan, M. Ghatee, and B. Hashemi, "Inverse of a fuzzy matrix of fuzzy numbers," International Journal of Computer Mathematics, vol. 86, no. 8, pp. 1433-1452, 2009.

[29] V. Kreinovich, "Membership functions or $\alpha$-Cuts? Algorithmic (constructivist) analysis justifies an interval approach," Mathematical Problems of Computer Science, vol. 38, pp. 70-71, 2012.

[30] R. Zou, Y. Liu, L. Liu, and H. Guo, "REILP approach for uncertainty-based decision making in civil engineering," Journal of Computing in Civil Engineering, vol. 24, no. 4, pp. 357-364, 2010.

[31] U.S. Environmental Protection Agency (USEPA), "Municipal Solid Waste in the United States: 2009 Facts and Figures," 2009.

[32] Q. Huang, Q. Wang, L. Dong, B. Xi, and B. Zhou, "The current situation of solid waste management in China," Journal of Material Cycles and Waste Management, vol. 8, no. 1, pp. 63-69, 2006.

[33] D. C. Wilson, "Long-term planning for solid waste management," Waste Management \& Research, vol. 3, no. 1, pp. 203-216, 1985.

[34] N. van Hop, "Solving fuzzy (stochastic) linear programming problems using superiority and inferiority measures," Information Sciences, vol. 177, no. 9, pp. 1977-1991, 2007. 


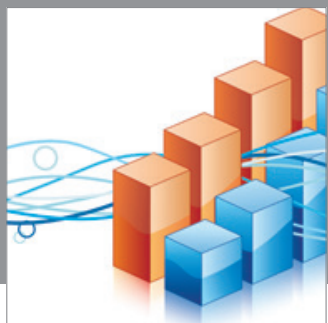

Advances in

Operations Research

mansans

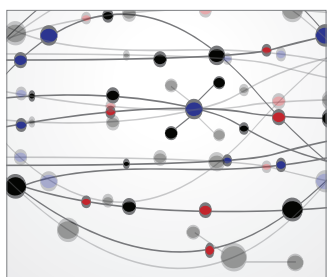

The Scientific World Journal
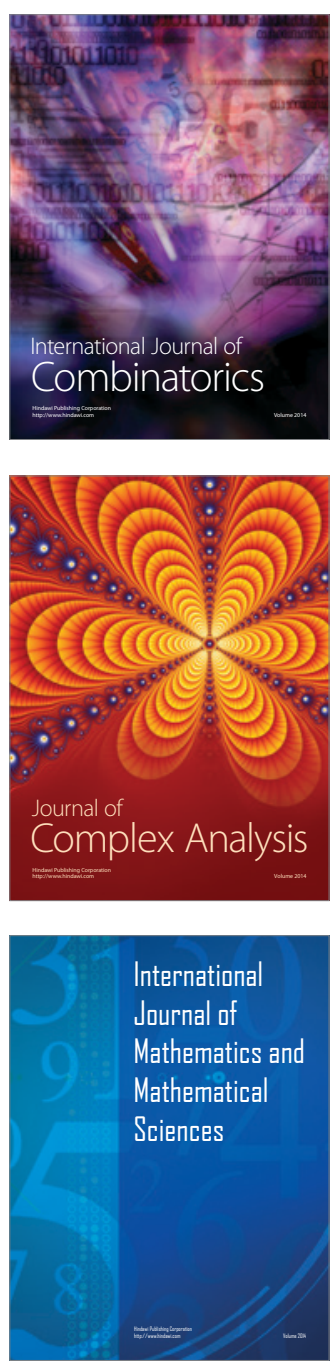
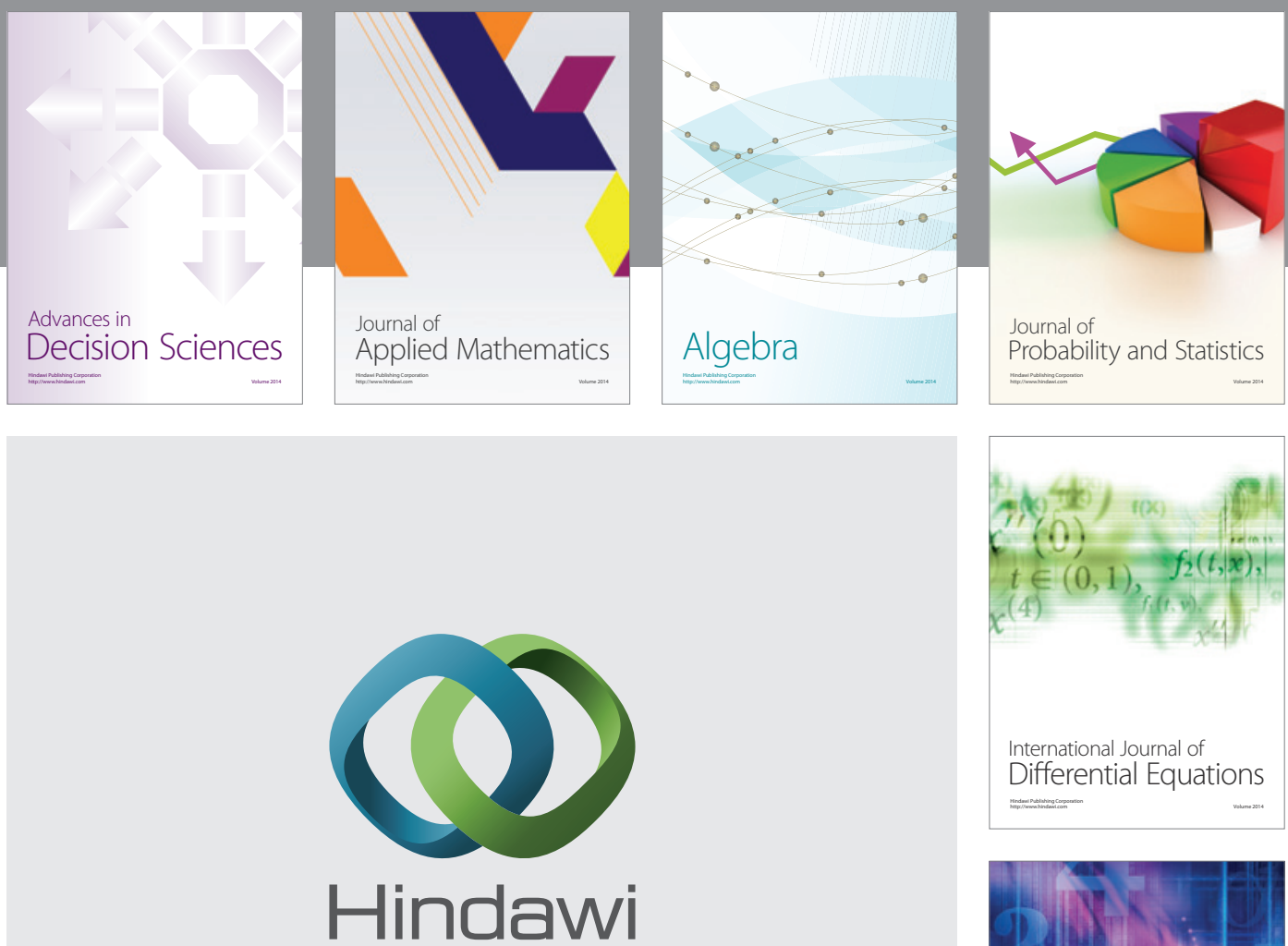

Submit your manuscripts at http://www.hindawi.com
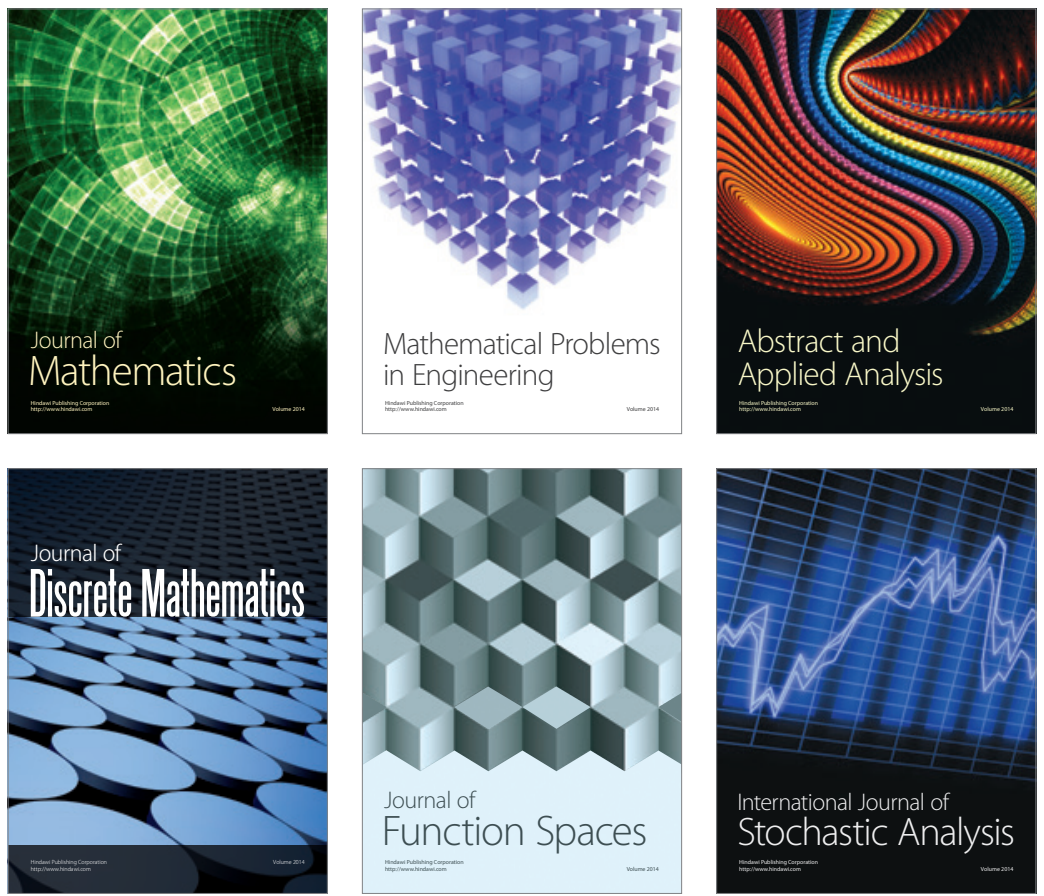

Journal of

Function Spaces

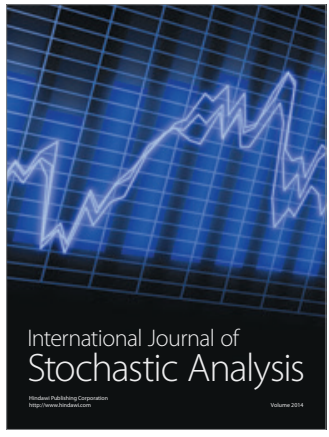

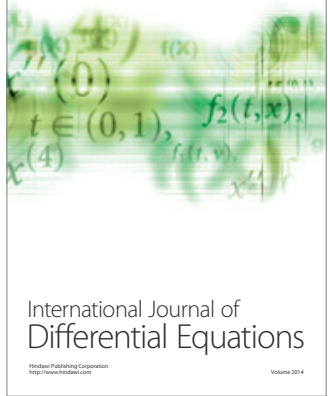
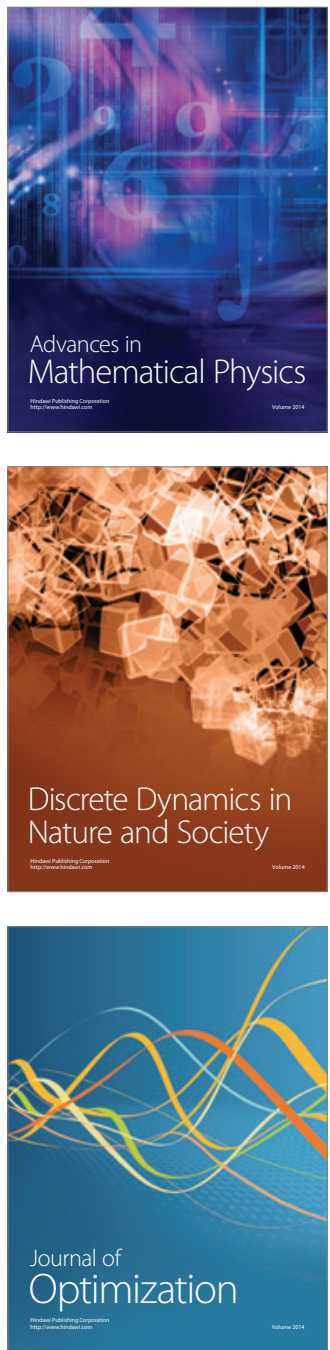